\title{
Infinite dimensional differential games with hybrid controls
}

\author{
A J SHAIJU and SHEETAL DHARMATTI* ${ }^{\dagger}$ \\ Department of Mathematics, Indian Institute of Science, Bangalore 560 012, India \\ *TIFR Centre, IISc Campus, Bangalore 560 012, India \\ E-mail: shaiju@math.tifrbng.res.in; sheetal@math.iisc.ernet.in \\ Corresponding author.
}

MS received 24 September 2005; revised 23 August 2006

\begin{abstract}
A two-person zero-sum infinite dimensional differential game of infinite duration with discounted payoff involving hybrid controls is studied. The minimizing player is allowed to take continuous, switching and impulse controls whereas the maximizing player is allowed to take continuous and switching controls. By taking strategies in the sense of Elliott-Kalton, we prove the existence of value and characterize it as the unique viscosity solution of the associated system of quasi-variational inequalities.
\end{abstract}

Keywords. Differential game; strategy; hybrid controls; value; viscosity solution.

\section{Introduction and preliminaries}

The study of differential games with Elliott-Kalton strategies in the viscosity solution framework is initiated by Evans and Souganidis [3] where both players are allowed to take continuous controls. Differential games where both players use switching controls are studied by Yong [6, 7]. In [8], differential games involving impulse controls are considered; one player is using continuous controls whereas the other uses impulse control. In the final section of [8], the author mentions that by using the ideas and techniques of the previous sections one can study differential games where one player uses continuous, switching and impulse controls and the other player uses continuous and switching controls. The uniqueness result for the associated system of quasi-variational inequalities (SQVI) with bilateral constraints is said to hold under suitable non-zero loop switching-cost condition and cheaper switching condition. In all the above references, the state space is a finitedimensional Euclidean space.

The infinite dimensional analogue of [3] is studied by Kocan et al [4], where the authors prove the existence of value and characterize the value function as the unique viscosity solution (in the sense of [2]) of the associated Hamilton-Jacobi-Isaacs equation.

In this paper, we study a two-person zero-sum differential game in a Hilbert space where the minimizer (player 2) uses three types of controls: continuous, switching and impulse. The maximizer (player 1) uses continuous and switching controls. We first prove dynamic programming principle (DPP) for this problem. Using DPP, we prove that the lower and upper value functions are 'approximate solutions' of the associated SQVI in the viscosity sense [2]. Finally we establish the existence of the value by proving a uniqueness theorem for SQVI. We obtain our results without any assumption like non-zero loop switchingcost condition and/or cheaper switching-cost condition on the cost functions. This will be further explained in the concluding section. Thus this paper not only generalises the results 
of [8] to the infinite dimensional state space, it obtains the main result under fairly general conditions as well.

The rest of the paper is organized as follows. We set up necessary notations and assumptions in the remaining part of this section. The statement of the main result is also given at the end of this introductory section. The DPP is proved in $\$ 2$. In this section we also show that the lower/upper value function is an 'approximate viscosity solution' of SQVI. Section 3 is devoted to the proof of the main uniqueness result for SQVI and the existence of value. We conclude the paper in $\$ 4$ with a few remarks.

We first describe the notations and basic assumptions. The state space is a separable Hilbert space $\mathbb{E}$. The continuous control set for player $i, i=1,2$, is $U^{i}$, a compact metric space. The set $D^{i}=\left\{d_{1}^{i}, \ldots, d_{m_{i}}^{i}\right\} ; i=1,2$; is the switching control set for player $i$. The impulse control set for the player 2 is $K$, a closed and convex subset of the state space $\mathbb{E}$. The space of all $U^{i}$-valued measurable maps on $[0, \infty)$ is the continuous control space for player $i$ and is denoted by $\mathcal{U}^{i}$ :

$$
\mathcal{U}^{i}=\left\{u^{i}:[0, \infty) \rightarrow U^{i} \mid u^{i} \text { measurable }\right\}
$$

By $\mathcal{U}^{i}[0, t]$ we mean the space of all $U^{i}$-valued measurable maps on $[0, t]$ that is,

$$
\mathcal{U}^{i}[0, t]=\left\{u^{i}:[0, t] \rightarrow U^{i} \mid u^{i} \text { measurable }\right\} .
$$

The switching control space $\mathcal{D}^{i}$ for player $i$ and the impulse control space $\mathcal{K}$ for player 2 are defined as follows:

$$
\begin{gathered}
\mathcal{D}^{i}=\left\{d^{i}(\cdot)=\sum_{j \geq 1} d_{j-1}^{i} \chi_{\left[\theta_{j-1}^{i}, \theta_{j}^{i}\right)}(\cdot): d_{j}^{i} \in D^{i},\left(\theta_{j}^{i}\right) \subset[0, \infty],\right. \\
\left.\theta_{0}^{i}=0,\left(\theta_{j}^{i}\right) \uparrow \infty, d_{j-1}^{i} \neq d_{j}^{i} \text { if } \theta_{j}^{i}<\infty\right\}, \\
\mathcal{K}=\left\{\xi(\cdot)=\sum_{j \geq 0} \xi_{j} \chi_{\left[\tau_{j}, \infty\right]}(\cdot): \xi_{j} \in K,\left(\tau_{j}\right) \subset[0, \infty],\left(\tau_{j}\right) \uparrow \infty\right\} .
\end{gathered}
$$

An impulse control $\xi(\cdot)=\sum_{j \geq 0} \xi_{j} \chi_{\left[\tau_{j}, \infty\right]}(\cdot)$, consists of the impulse times $\tau_{j}$ 's and impulse vectors $\xi_{j}$ 's. We use the notation

$$
(\xi)_{1, j}=\tau_{j} \text { and }(\xi)_{2, j}=\xi_{j}
$$

Similarly for switching controls $d^{1}(\cdot)$ and $d^{2}(\cdot)$ we write

$$
\begin{aligned}
& \left(d^{1}\right)_{1, j}=\theta_{j}^{1} \quad \text { and } \quad\left(d^{1}\right)_{2, j}=d_{j}^{1}, \\
& \left(d^{2}\right)_{1, j}=\theta_{j}^{2} \quad \text { and } \quad\left(d^{2}\right)_{2, j}=d_{j}^{2} .
\end{aligned}
$$

Now we describe the dynamics and cost functions involved in the game. To this end, let $\mathcal{C}^{1}=\mathcal{U}^{1} \times \mathcal{D}^{1}$ and $\mathcal{C}^{2}=\mathcal{U}^{2} \times \mathcal{D}^{2} \times \mathcal{K}$. For $\left(u^{1}(\cdot), d^{1}(\cdot)\right) \in \mathcal{C}^{1}$ and $\left(u^{2}(\cdot), d^{2}(\cdot), \xi(\cdot)\right) \in \mathcal{C}^{2}$, the corresponding state $y_{x}(\cdot)$ is governed by the following controlled semilinear evolution equation in $\mathbb{E}$ :

$$
\dot{y}_{x}(t)+A y_{x}(t)=f\left(y_{x}(t), u^{1}(t), d^{1}(t), u^{2}(t), d^{2}(t)\right)+\dot{\xi}(t), \quad y_{x}(0-)=x,
$$


where $f: \mathbb{E} \times U^{1} \times D^{1} \times U^{2} \times D^{2} \rightarrow \mathbb{E}$ and $-A$ is the generator of a contraction semigroup $\{S(t) ; t \geq 0\}$ on $\mathbb{E}$.

(A1) We assume that the function $f$ is bounded, continuous and for all $x, y \in \mathbb{E}, d^{i} \in D^{i}$, $u^{i} \in U^{i}$,

$$
\left\|f\left(x, u^{1}, d^{1}, u^{2}, d^{2}\right)-f\left(y, u^{1}, d^{1}, u^{2}, d^{2}\right)\right\| \leq L\|x-y\| .
$$

Note that under the assumption (A1), for each $x \in \mathbb{E}, d^{i}(\cdot) \in \mathcal{D}^{i}, u^{i}(\cdot) \in \mathcal{U}^{i}$ and $\xi(\cdot) \in \mathcal{K}$ there is a unique mild solution $y_{x}(\cdot)$ of $(1.1)$. This can be concluded for example, from Corollary 2.11, chapter 4, page number 109 of [5].

Let $k: \mathbb{E} \times U^{1} \times D^{1} \times U^{2} \times D^{2} \rightarrow \mathbb{R}$ be the running cost function, $c^{i}: D^{i} \times D^{i} \rightarrow \mathbb{R}$ the switching cost functions, and $l: K \rightarrow \mathbb{R}$ the impulse cost function.

(A2) We assume that the cost functions $k, c^{i}, l$ are nonnegative, bounded, continuous, and for all $x, y \in \mathbb{E}, d^{i} \in D^{i}, u^{i} \in U^{i}, \xi_{0}, \xi_{1} \in K$,

$$
\begin{aligned}
\left|k\left(x, u^{1}, d^{1}, u^{2}, d^{2}\right)-k\left(y, u^{1}, d^{1}, u^{2}, d^{2}\right)\right| & \leq L\|x-y\|, \\
l\left(\xi_{0}+\xi_{1}\right) & <l\left(\xi_{0}\right)+l\left(\xi_{1}\right), \forall \xi_{0}, \xi_{1} \in K, \\
\lim _{|\xi| \rightarrow \infty} l(\xi) & =\infty, \\
\inf _{d_{1}^{i} \neq d_{2}^{i}} c^{i}\left(d_{1}^{i}, d_{2}^{i}\right) & =c_{0}^{i}>0 .
\end{aligned}
$$

Remark 1.1. The subadditivity condition $l\left(\xi_{0}+\xi_{1}\right)<l\left(\xi_{0}\right)+l\left(\xi_{1}\right)$ is needed to prove Lemma 3.1 which, in turn, is required to establish the uniqueness theorems (and hence the existence of value for the game) in $\S 3$. This condition makes sure that, if an impulse $\xi_{0}$ is the best option at a particular state $y_{0}$, then applying an impulse again is not a good option for the new state $y_{0}+\xi_{0}$.

Let $\lambda>0$ be the discount parameter. The total discounted cost functional $J_{x}: \mathcal{C}^{1} \times \mathcal{C}^{2} \rightarrow$ $\mathbb{R}$ is given by

$$
\left.\begin{array}{rl}
J_{x}\left[u^{1}(\cdot), d^{1}(\cdot), u^{2}(\cdot), d^{2}(\cdot), \xi(\cdot)\right]= & \int_{0}^{\infty} \mathrm{e}^{-\lambda t} k\left(y_{x}(t), u^{1}(t), d^{1}(t), u^{2}(t), d^{2}(t)\right) \mathrm{d} t \\
& -\sum_{j \geq 0} \mathrm{e}^{-\lambda \theta_{j}^{1}} c^{1}\left(d_{j-1}^{1}, d_{j}^{1}\right) \\
& +\sum_{j \geq 0} \mathrm{e}^{-\lambda \theta_{j}^{2}} c^{2}\left(d_{j-1}^{2}, d_{j}^{2}\right)+\sum_{j \geq 1} \mathrm{e}^{-\lambda \tau_{j}} l\left(\xi_{j}\right)
\end{array}\right\}
$$

We next define the strategies for player 1 and player 2 in the Elliott-Kalton framework. The strategy set $\Gamma$ for player 1 is the collection of all nonanticipating maps $\alpha$ from $\mathcal{C}^{2}$ to $\mathcal{C}^{1}$. The strategy set $\Delta$ for player 2 is the collection of all nonanticipating maps $\beta$ from $\mathcal{C}^{1}$ to $\mathcal{C}^{2}$.

For a strategy $\beta$ of player 2 if $\beta\left(u^{1}(\cdot), d^{1}(\cdot)\right)=\left(u^{2}(\cdot), d^{2}(\cdot), \xi(\cdot)\right)$, then we write $\Pi_{1} \beta\left(u^{1}(\cdot), d^{1}(\cdot)\right)=u^{2}(\cdot), \Pi_{2} \beta\left(u^{1}(\cdot), d^{1}(\cdot)\right)=d^{2}(\cdot)$ and $\Pi_{3} \beta\left(u^{1}(\cdot), d^{1}(\cdot)\right)=\xi(\cdot)$. 
That is, $\Pi_{i}$ is the projection on the $i$ th component of the map $\beta$. Similar notations are used for $\alpha\left(u^{2}(\cdot), d^{2}(\cdot), \xi(\cdot)\right)$ as well. Hence,

$$
\Pi_{1} \alpha\left(u^{2}(\cdot), d^{2}(\cdot), \xi(\cdot)\right)=u^{1}(\cdot) \quad \text { and } \quad \Pi_{2} \alpha\left(u^{2}(\cdot), d^{2}(\cdot), \xi(\cdot)\right)=d^{1}(\cdot) .
$$

Let $\mathcal{D}^{i, d^{i}}$ denote the set of all switching controls for player $i$ starting at $d^{i}$. Then we define sets

$$
\mathcal{C}^{1, d^{1}}=\mathcal{U}^{1} \times \mathcal{D}^{1, d^{1}} \quad \text { and } \quad \mathcal{C}^{2, d^{2}}=\mathcal{U}^{2} \times \mathcal{D}^{2, d^{2}} \times \mathcal{K} .
$$

Let $\Delta^{d^{2}}$ denote the collection of all $\beta \in \Delta$ such that $\Pi_{2} \beta(0)=d^{2}$ and $\Gamma^{d^{1}}$ be the collection of all $\alpha \in \Gamma$ such that $\Pi_{2} \alpha(0)=d^{1}$.

Now using these strategies we define upper and lower value functions associated with the game. Consider $J_{x}$ as defined in (1.4). Let $J_{x}^{d^{1}, d^{2}}$ be the restriction of the cost functional $J_{x}$ to $\mathcal{C}^{1, d^{1}} \times \mathcal{C}^{2, d^{2}}$. The upper and lower value functions are defined respectively as follows:

$$
\begin{aligned}
& V_{+}^{d^{1}, d^{2}}(x)=\sup _{\alpha \in \Gamma^{d^{1}}} \inf _{\mathcal{C}^{2, d^{2}}} J_{x}^{d^{1}, d^{2}}\left[\alpha\left(u^{2}(\cdot), d^{2}(\cdot), \xi(\cdot)\right), u^{2}(\cdot), d^{2}(\cdot), \xi(\cdot)\right], \\
& V_{-}^{d^{1}, d^{2}}(x)=\inf _{\beta \in \Delta^{d^{2}}} \sup _{\mathcal{C}^{1, d^{1}}} J_{x}^{d^{1}, d^{2}}\left[u^{1}(\cdot), d^{1}(\cdot), \beta\left(u^{1}(\cdot), d^{1}(\cdot)\right)\right] .
\end{aligned}
$$

Let $V_{+}=\left\{V_{+}^{d^{1}, d^{2}}:\left(d^{1}, d^{2}\right) \in D^{1} \times D^{2}\right\}$ and $V_{-}=\left\{V_{-}^{d^{1}, d^{2}}:\left(d^{1}, d^{2}\right) \in D^{1} \times D^{2}\right\}$. If $V_{+} \equiv V_{-} \equiv V$, then we say that the differential game has a value and $V$ is referred to as the value function.

Since all cost functions involved are bounded, value functions are also bounded. In view of (A1) and (A2), the proof of uniform continuity of $V_{+}$and $V_{-}$is routine. Hence both $V_{+}$ and $V_{-}$belong to BUC $\left(\mathbb{E} ; \mathbb{R}^{m_{1} \times m_{2}}\right)$, the space of bounded uniformly continuous functions from $\mathbb{E}$ to $\mathbb{R}^{m_{1} \times m_{2}}$.

Now we describe the system of quasivariational inequalities (SQVI) satisfied by upper and lower value functions and the definition of viscosity solution in the sense of [2].

For $x, p \in \mathbb{E}$, let

$$
\begin{aligned}
& H_{-}^{d^{1}, d^{2}}(x, p)=\max _{u^{2} \in U^{2}} \min _{u^{1} \in U^{1}}\left[\left\langle-p, f\left(x, u^{1}, d^{1}, u^{2}, d^{2}\right)\right\rangle-k\left(x, u^{1}, d^{1}, u^{2}, d^{2}\right)\right], \\
& H_{+}^{d^{1}, d^{2}}(x, p)=\min _{u^{1} \in U^{1}} \max _{u^{2} \in U^{2}}\left[\left\langle-p, f\left(x, u^{1}, d^{1}, u^{2}, d^{2}\right)\right\rangle-k\left(x, u^{1}, d^{1}, u^{2}, d^{2}\right)\right]
\end{aligned}
$$

and for $V \in C\left(\mathbb{E} ; \mathbb{R}^{m_{1} \times m_{2}}\right)$, let

$$
\begin{aligned}
& M_{-}^{d^{1}, d^{2}}[V](x)=\min _{\bar{d}^{2} \neq d^{2}}\left[V^{d^{1}, \bar{d}^{2}}(x)+c^{2}\left(d^{2}, \bar{d}^{2}\right)\right], \\
& M_{+}^{d^{1}, d^{2}}[V](x)=\max _{\bar{d}^{1} \neq d^{1}}\left[V^{\bar{d}^{1}, d^{2}}(x)-c^{1}\left(d^{1}, \bar{d}^{1}\right)\right], \\
& N\left[V^{d^{1}, d^{2}}\right](x)=\inf _{\xi \in K}\left[V^{d^{1}, d^{2}}(x+\xi)+l(\xi)\right] .
\end{aligned}
$$


The HJI upper systems of equations associated with $V_{-}$of the hybrid differential game are as follows: for $\left(d^{1}, d^{2}\right) \in D^{1} \times D^{2}$,

$$
\begin{aligned}
& \min \left\{\operatorname { m a x } \left(\lambda V^{d^{1}, d^{2}}+\left\langle A x, D V^{d^{1}, d^{2}}\right\rangle+H_{+}^{d^{1}, d^{2}}\left(x, D V^{d^{1}, d^{2}}\right)\right.\right. \\
& \left.\left.\left.V^{d^{1}, d^{2}}-M_{-}^{d^{1}, d^{2}}[V], V^{d^{1}, d^{2}}-N\left[V^{d^{1}, d^{2}}\right]\right), V^{d^{1}, d^{2}}-M_{+}^{d^{1}, d^{2}}[V]\right\}=0\right\}, \\
& \left.\begin{array}{l}
\max \left\{\operatorname { m i n } \left(\lambda V^{d^{1}, d^{2}}+\left\langle A x, D V^{d^{1}, d^{2}}\right\rangle+H_{+}^{d^{1}, d^{2}}\left(x, D V^{d^{1}, d^{2}}\right),\right.\right. \\
\left.\left.V^{d^{1}, d^{2}}-M_{+}^{d^{1}, d^{2}}[V]\right), \quad V^{d^{1}, d^{2}}-M_{-}^{d^{1}, d^{2}}[V], V^{d^{1}, d^{2}}-N\left[V^{d^{1}, d^{2}}\right]\right\}=0
\end{array}\right\},
\end{aligned}
$$

where $H_{+}^{d^{1}, d^{2}}, M_{-}^{d^{1}, d^{2}}, M_{+}^{d^{1}, d^{2}}$ and $N\left[V^{d^{1}, d^{2}}\right]$ are as given by (1.8), (1.9), (1.10) and (1.11) respectively.

Note here that for any real numbers $a, b, c, d,(a \vee b) \wedge c \wedge d \leq(a \vee c \vee d) \wedge b$.

If we replace $H_{+}^{d^{1}, d^{2}}$ in the above system of equations by $H_{-}^{d^{1}, d^{2}}$, then we obtain the HJI lower system of equations associated with $V_{+}$and is denoted respectively by (HJI1-) and (HJI2-).

If $V$ satisfies both (HJI1+) and (HJI2+), then we say that $V$ satisfies (HJI+) and similarly if it satisfies both (HJI-) and (HJI2-), we say that $V$ satisfies (HJI-) .

Now let us recall the definition of viscosity solution (in the sense of Crandall and Lions [2]). To this end, let

$$
\begin{aligned}
C^{1}(\mathbb{E}) & =\{\phi: \mathbb{E} \rightarrow \mathbb{R} \mid \phi \text { continuously differentiable }\}, \\
\operatorname{Lip}(\mathbb{E}) & =\{\psi: \mathbb{E} \rightarrow \mathbb{R} \mid \psi \text { Lipschitz continuous }\}, \\
\mathcal{T} & =\left\{\Phi \mid \Phi=\phi+\psi, \phi \in C^{1}(\mathbb{E}), \psi \in \operatorname{Lip}(\mathbb{E})\right\}, \\
D_{A}^{+} \Phi(x) & =\limsup _{\delta \downarrow 0, y \rightarrow x} \frac{1}{\delta}[\Phi(y)-\Phi(S(\delta) y)], \\
D_{A}^{-} \Phi(x) & =\liminf _{\delta \downarrow 0, y \rightarrow x} \frac{1}{\delta}[\Phi(y)-\Phi(S(\delta) y)], \\
H_{+, \frac{\bar{r}}{d^{1}}, d^{2}}(x, p) & =\sup _{\|q\| \leq r} H_{+}^{d^{1}, d^{2}}(x, p+q) ; \quad H_{-, \bar{r}}^{d^{1}, d^{2}}(x, p)=\sup _{\|q\| \leq r} H_{-}^{d^{1}, d^{2}}(x, p+q) ; \\
H_{+, \underline{r}}^{d^{1}, d^{2}}(x, p) & =\inf _{\|q\| \leq r} H_{+}^{d^{1}, d^{2}}(x, p+q) ; \quad H_{-, \underline{r}}^{d^{1}, d^{2}}(x, p)=\inf _{\|q\| \leq r} H_{-}^{d^{1}, d^{2}}(x, p+q) .
\end{aligned}
$$

\section{DEFINITION 1.2}

A continuous function $V$ is a viscosity subsolution of $(\mathrm{HJI} 1+)$ if

$$
\begin{gathered}
\min \left\{\operatorname { m a x } \left(\lambda V^{d^{1}, d^{2}}(\hat{x})+D_{A}^{-} \Phi(\hat{x})+H_{\left.+, \underline{d^{1}}, d^{2}\right)}(\hat{x}, D \phi(\hat{x})), V^{d^{1}, d^{2}}(\hat{x})-M_{-}^{d^{1}, d^{2}}[V](\hat{x}),\right.\right. \\
\left.\left.V^{d^{1}, d^{2}}(\hat{x})-N\left[V^{d^{1}, d^{2}}\right](\hat{x})\right), V^{d^{1}, d^{2}}(\hat{x})-M_{+}^{d^{1}, d^{2}}[V](\hat{x})\right\} \leq 0,
\end{gathered}
$$

for any $\Phi \in \mathcal{T},\left(d^{1}, d^{2}\right) \in D^{1} \times D^{2}$ and local maximum $\hat{x}$ of $V^{d^{1}, d^{2}}-\Phi$. 
A continuous function $V$ is a viscosity supersolution of (HJI1+) if

$$
\begin{aligned}
& \min \left\{\operatorname { m a x } \left(\lambda V^{d^{1}, d^{2}}(\hat{x})+D_{A}^{+} \Phi(\hat{x})+H_{+, \frac{d^{1}}{,} d^{2}}^{L(\psi)}(\hat{x}, D \phi(\hat{x})), V^{d^{1}, d^{2}}(\hat{x})-M_{-}^{d^{1}, d^{2}}[V](\hat{x}),\right.\right. \\
& \left.\left.V^{d^{1}, d^{2}}(\hat{x})-N\left[V^{d^{1}, d^{2}}\right](\hat{x})\right), V^{d^{1}, d^{2}}(\hat{x})-M_{+}^{d^{1}, d^{2}}[V](\hat{x})\right\} \geq 0,
\end{aligned}
$$

for any $\Phi \in \mathcal{T},\left(d^{1}, d^{2}\right) \in D^{1} \times D^{2}$ and local minimum $\hat{x}$ of $V^{d^{1}, d^{2}}-\Phi$.

If $V$ is both a subsolution and a supersolution of (HJI1+), then we say that $V$ is a viscosity solution of (HJI1+).

\section{DEFINITION 1.3}

A continuous function $V$ is an approximate viscosity subsolution of (HJI1+) if for all $R>0$, there exists a constant $C_{R}>0$ such that

$$
\begin{aligned}
& \min \left\{\operatorname { m a x } \left(\lambda V^{d^{1}, d^{2}}(\hat{x})+D_{A}^{-} \Phi(\hat{x})+H_{+}^{d^{1}, d^{2}}\left(\hat{x}, D \phi(\hat{x})-C_{R} L(\psi)\right),\right.\right. \\
& \left.\left.\quad V^{d^{1}, d^{2}}(\hat{x})-M_{-}^{d^{1}, d^{2}}[V](\hat{x}), V^{d^{1}, d^{2}}(\hat{x})-N\left[V^{d^{1}, d^{2}}\right](\hat{x})\right), V^{d^{1}, d^{2}}(\hat{x})-M_{+}^{d^{1}, d^{2}}[V](\hat{x})\right\} \\
& \leq 0,
\end{aligned}
$$

for any $\Phi \in \mathcal{T},\left(d^{1}, d^{2}\right) \in D^{1} \times D^{2}$ and local maximum $\hat{x} \in B_{R}(0)$ of $V^{d^{1}, d^{2}}-\Phi$.

A continuous function $V$ is an approximate viscosity supersolution of (HJI1+) if for all $R>0$, there exists a constant $C_{R}>0$ such that

$$
\begin{aligned}
\min & \left\{\operatorname { m a x } \left(\lambda V^{d^{1}, d^{2}}(\hat{x})+D_{A}^{+} \Phi(\hat{x})+H_{+}^{d^{1}, d^{2}}\left(\hat{x}, D \phi(\hat{x})+C_{R} L(\psi)\right),\right.\right. \\
& \left.\left.V^{d^{1}, d^{2}}(\hat{x})-M_{-}^{d^{1}, d^{2}}[V](\hat{x}), V^{d^{1}, d^{2}}(\hat{x})-N\left[V^{d^{1}, d^{2}}\right](\hat{x})\right), V^{d^{1}, d^{2}}(\hat{x})-M_{+}^{d^{1}, d^{2}}[V](\hat{x})\right\} \\
\geq 0, &
\end{aligned}
$$

for any $\Phi \in \mathcal{T},\left(d^{1}, d^{2}\right) \in D^{1} \times D^{2}$ and local minimum $\hat{x} \in B_{R}(0)$ of $V^{d^{1}, d^{2}}-\Phi$.

If $V$ is both an approximate subsolution and an approximate supersolution of (HJI1+), then we say that $V$ is an approximate viscosity solution of $(\mathrm{HJI} 1+)$.

In the above definitions, $L(\psi)$ is the Lipschitz constant of $\psi$ and $B_{R}(0)$ is the closed ball of radius $R$ around the origin.

Remark 1.4. One can easily prove that a viscosity solution is always an approximate viscosity solution. For more details about the approximate viscosity solution and its connections with other notions of solutions, we refer to [2] and [4]. In the infinite dimensional set-up it is easier to establish that the value functions are approximate viscosity solutions than to prove that they are viscosity solutions. Therefore, as pointed out in [4], the concept of approximate viscosity solution is used as a vehicle to prove that the value functions are viscosity solutions.

In the next section, we show that $V_{-}$is an approximate viscosity solution of (HJI+) and $V_{+}$is an approximate viscosity solution of (HJI-).

We say that the Isaacs min-max condition holds if

$$
H_{-}^{d^{1}, d^{2}} \equiv H_{+}^{d^{1}, d^{2}} \quad \text { for all }\left(d^{1}, d^{2}\right) \in D^{1} \times D^{2} .
$$


Under this condition, the equations (HJI1+) and (HJI2+) respectively coincide with (HJI1-) and (HJI2-). We now state the main result of this paper; the proof will be worked out in subsequent sections.

Theorem 1.5. Assume (A1), (A2) and the Isaacs min-max condition. Then $V_{-}=V_{+}$is the unique viscosity solution of $(\mathrm{HJI}+)$ (or $(\mathrm{HJI}-))$ in $\mathrm{BUC}\left(\mathbb{E}, \mathbb{R}^{m_{1} \times m_{2}}\right)$.

Remark 1.6. The Isaacs min-max condition (1.12) holds for the class of problems where $f$ is of the form

$$
f\left(x, u^{1}, d^{1}, u^{2}, d^{2}\right)=f_{1}\left(x, u^{1}, d^{1}, d^{2}\right)+f_{2}\left(x, d^{1}, u^{2}, d^{2}\right) .
$$

\section{Dynamic programming principle}

In this section, we first prove the dynamic programming principle for the differential games with hybrid controls. We first state the results. The proofs will be given later. Throughout this section we assume (A1) and (A2).

Lemma 2.1. For $\left(x, d^{1}, d^{2}\right) \in \mathbb{E} \times D^{1} \times D^{2}$ and $t>0$,

$$
\left.\begin{array}{rl}
V_{-}^{d^{1}, d^{2}}(x)= & \inf _{\beta \in \Delta^{d^{2}}} \sup _{\mathcal{C}^{1, d^{1}}}\left[\int _ { 0 } ^ { t } \mathrm { e } ^ { - \lambda s } k \left(y_{x}(s), u^{1}(s), d^{1}(s),\right.\right. \\
& \left.\Pi_{1} \beta\left(u^{1}, d^{1}\right)(s), \Pi_{2} \beta\left(u^{1}, d^{1}\right)(s)\right) \mathrm{d} s \\
& -\sum_{\theta_{j}^{1}<t} \mathrm{e}^{-\lambda \theta_{j}^{1}} c^{1}\left(d_{j-1}^{1}, d_{j}^{1}\right) \\
& +\sum_{\left(\Pi_{2} \beta\right)_{1, j}<t} \mathrm{e}^{-\lambda\left(\Pi_{2} \beta\right)_{1, j}} c^{2}\left(\left(\Pi_{2} \beta\right)_{2, j-1},\left(\Pi_{2} \beta\right)_{2, j}\right) \\
& +\sum_{\left(\Pi_{3} \beta\right)_{1, j}<t} \mathrm{e}^{-\lambda\left(\Pi_{3} \beta\right)_{1, j}} l\left(\left(\Pi_{3} \beta\right)_{2, j}\right) \\
& \left.+\mathrm{e}^{-\lambda t} V_{-}^{d^{1}(t), \Pi_{2} \beta(t)}\left(y_{x}(t)\right)\right]
\end{array}\right\}
$$

Lemma 2.2. For $\left(x, d^{1}, d^{2}\right) \in \mathbb{E} \times D^{1} \times D^{2}$ and $t>0$,

$$
\begin{aligned}
V_{+}^{d^{1}, d^{2}}(x)= & \sup _{\alpha \in \Gamma^{d^{1}} \mathcal{C}^{2, d^{2}}}\left[\int_{0}^{t} \mathrm{e}^{-\lambda s} k\left(y_{x}(s), \alpha\left(u^{2}, d^{2}, \xi\right)(s), u^{2}(s), d^{2}(s), \xi(s)\right) \mathrm{d} s\right. \\
& -\sum_{\left(\Pi_{2} \alpha\right)_{1, j}<t} \mathrm{e}^{-\lambda\left(\Pi_{2} \alpha\right)_{1, j}} c^{1}\left(\left(\Pi_{2} \alpha\right)_{1, j-1},\left(\Pi_{2} \alpha\right) 1, j\right) \\
& +\sum_{\theta_{j}^{2}<t} \mathrm{e}^{-\lambda \theta_{j}^{2}} c^{2}\left(d_{j-1}^{2}, d_{j}^{2}\right) \\
& \left.+\sum_{\tau_{j}<t} \mathrm{e}^{-\lambda \tau_{j}} l\left(\xi_{j}\right)+\mathrm{e}^{-\lambda t} V_{+}^{\Pi_{2} \alpha(t), d^{2}(t)}\left(y_{x}(t)\right)\right] .
\end{aligned}
$$


Lemma 2.3. The following results hold:

(i) $M_{+}^{d^{1}, d^{2}}\left[V_{-}\right](x) \leq V_{-}^{d^{1}, d^{2}}(x)$.

(ii) $V_{-}^{d^{1}, d^{2}}(x) \leq \min \left\{M_{-}^{d^{1}, d^{2}}\left[V_{-}\right](x), N\left[V_{-}^{d^{1}, d^{2}}\right](x)\right\}$.

(iii) Let $\left(x, d^{1}, d^{2}\right)$ be such that strict inequality holds in (i). Let $\bar{\beta} \in \Delta_{0}^{d^{2}}$. Then there exists $t_{0}>0$ such that the following holds:

For each $0 \leq t \leq t_{0}$, there exists $u^{1, t}(\cdot) \in \mathcal{U}^{1}[0, t]$ such that

$$
\begin{aligned}
V_{-}^{d^{1}, d^{2}}(x)-t^{2} \leq & \int_{0}^{t} \mathrm{e}^{-\lambda s} k\left(y_{x}(s), u^{1, t}(s), d^{1}, \bar{\beta}\left(u^{1, t}(\cdot), d^{1}\right)(s)\right) \mathrm{d} s \\
& +\mathrm{e}^{-\lambda t} V_{-}^{d^{1}, d^{2}}\left(y_{x}(t)\right) .
\end{aligned}
$$

(iv) Let $\left(x, d^{1}, d^{2}\right)$ be such that strict inequality holds in (ii). Let $\bar{u}^{1} \in U^{1}$. Then there exists $t_{0}>0$ such that the following holds:

For each $0 \leq t \leq t_{0}$, there exists $\beta^{t} \in \Delta^{d^{2}}$ with $\left(\Pi_{2} \beta^{t}\left(\bar{u}^{1}, d^{1}\right)\right)_{1,1},\left(\Pi_{3} \beta^{t}\left(\bar{u}^{1}, d^{1}\right)\right)_{1,1}>$ $t_{0}$ such that

$$
\begin{aligned}
V_{-}^{d^{1}, d^{2}}(x)+t^{2} \geq & \int_{0}^{t} \mathrm{e}^{-\lambda s} k\left(y_{x}(s), \bar{u}^{1}, d^{1}, \beta^{t}\left(\bar{u}^{1}, d^{1}\right)(s)\right) \mathrm{d} s \\
& +\mathrm{e}^{-\lambda t} V_{-}^{d^{1}, d^{2}}\left(y_{x}(t)\right) .
\end{aligned}
$$

\section{Lemma 2.4. The following results hold.}

(i) $M_{+}^{d^{1}, d^{2}}\left[V_{+}\right](x) \leq V_{+}^{d^{1}, d^{2}}(x)$.

(ii) $V_{+}^{d^{1}, d^{2}}(x) \leq \min \left\{M_{-}^{d^{1}, d^{2}}\left[V_{+}\right](x), N\left[V_{+}^{d^{1}, d^{2}}\right](x)\right\}$.

(iii) Let $\left(x, d^{1}, d^{2}\right)$ be such that strict inequality holds in (ii). Let $\bar{\alpha} \in \Gamma_{0}^{d^{1}}$. Then there exists $t_{0}>0$ such that the following holds:

For each $0 \leq t \leq t_{0}$, there exists $u^{2, t}(\cdot) \in \mathcal{U}^{2}[0, t]$ such that

$$
\begin{aligned}
V_{+}^{d^{1}, d^{2}}(x)+t^{2} \geq & \int_{0}^{t} \mathrm{e}^{-\lambda s} k\left(y_{x}(s), u^{2, t}(s), d^{2}, \bar{\alpha}\left(u^{2, t}, d^{2}, \xi^{\infty}\right)(s)\right) \mathrm{d} s \\
& +\mathrm{e}^{-\lambda t} V_{+}^{d^{1}, d^{2}}\left(y_{x}(t)\right) .
\end{aligned}
$$

(iv) Let $\left(x, d^{1}, d^{2}\right)$ be such that strict inequality holds in (i). Let $\bar{u}^{2} \in U^{2}$. Then there exists $t_{0}>0$ such that the following holds:

For each $0 \leq t \leq t_{0}$, there exists $\alpha^{t} \in \Gamma^{d^{1}}$ with $\left(\Pi_{2} \alpha^{t}\left(\bar{u}^{2}, d^{2}, \xi^{\infty}\right)\right)_{1,1}>t_{0}$ such that

$$
\begin{aligned}
V_{+}^{d^{1}, d^{2}}(x)-t^{2} \leq & \int_{0}^{t} \mathrm{e}^{-\lambda s} k\left(y_{x}(s), \bar{u}^{2}, d^{2}, \alpha^{t}\left(\bar{u}^{2}, d^{2}, \xi^{\infty}\right)(s)\right) \mathrm{d} s \\
& +\mathrm{e}^{-\lambda t} V_{+}^{d^{1}, d^{2}}\left(y_{x}(t)\right)
\end{aligned}
$$

We prove Lemmas 2.1 and 2.3. The proofs of Lemmas 2.2 and 2.4 are analogous. 
Proof of Lemma 2.1. Let $\left(x, d^{1}, d^{2}\right) \in \mathbb{E} \times D^{1} \times D^{2}$ and $t>0$. Let us denote the RHS of (2.1) by $W(x)$. Fix $\epsilon>0$.

Let $\bar{\beta} \in \Delta^{d^{2}}$ be such that

$$
\begin{aligned}
W(x) \geq & \sup _{\mathcal{C}^{1, d^{1}}}\left[\int_{0}^{t} \mathrm{e}^{-\lambda s} k\left(y_{x}(s), u^{1}(s), d^{1}(s), \Pi_{1} \bar{\beta}\left(u^{1}, d^{1}\right)(s), \Pi_{2} \bar{\beta}\left(u^{1}, d^{1}\right)(s)\right) \mathrm{d} s\right. \\
& -\sum_{\theta_{j}^{1}<t} \mathrm{e}^{-\lambda \theta_{j}^{1}} c^{1}\left(d_{j-1}^{1}, d_{j}^{1}\right)+\sum_{\left(\Pi_{2} \bar{\beta}\right)_{1, j}<t} \mathrm{e}^{-\lambda\left(\Pi_{2} \bar{\beta}\right)_{1, j}} c^{2}\left(\left(\Pi_{2} \bar{\beta}\right)_{2, j-1},\left(\Pi_{2} \bar{\beta}\right)_{2, j}\right) \\
& \left.+\sum_{\left(\Pi_{3} \bar{\beta}\right)_{1, j}<t} \mathrm{e}^{-\lambda\left(\Pi_{3} \bar{\beta}\right)_{1, j}} l\left(\left(\Pi_{3} \bar{\beta}\right)_{2, j}\right)+\mathrm{e}^{-\lambda t} V_{-}^{d^{1}(t),\left(\Pi_{2} \bar{\beta}\right)(t)}\left(y_{x}(t)\right)\right]-\epsilon .
\end{aligned}
$$

By the definition of $V_{-}$, for each $\left(u^{1}(\cdot), d^{1}(\cdot)\right) \in \mathcal{C}^{1, d^{1}}$, there exists $\beta_{u^{1}(\cdot), d^{1}(\cdot)} \in$ $\Delta^{\left(\Pi_{2} \bar{\beta}\right)(t)}$ such that

$$
\begin{aligned}
& V_{-}^{d^{1}(t),\left(\Pi_{2} \bar{\beta}\right)(t)}\left(y_{x}(t)\right) \\
& \quad \geq J_{y_{x}(t)}^{d^{1}(t),\left(\left(\Pi_{2} \bar{\beta}\right)(t)\right.}\left[u^{1}(\cdot), d^{1}(\cdot), \beta_{u^{1}(\cdot), d^{1}(\cdot)}\left(u^{1}(\cdot), d^{1}(\cdot)\right)\right]-\epsilon .
\end{aligned}
$$

Define $\delta \in \Delta^{d^{2}}$ by

$$
\delta\left(u^{1}(\cdot), d^{1}(\cdot)\right)(s)=\left\{\begin{array}{ll}
\bar{\beta}\left(u^{1}(\cdot), d^{1}(\cdot)\right)(s) ; & s \leq t \\
\beta_{u^{1}(\cdot), d^{1}(\cdot)}\left(u^{1}(\cdot+t), d^{1}(\cdot+t)\right)(s-t) ; & s>t
\end{array} .\right.
$$

By change of variables, we get

$$
\begin{aligned}
& J_{y_{x}(t)}^{d^{1}(t),\left(\Pi_{2} \bar{\beta}\right)(t)}\left[u^{1}(\cdot+t), d^{1}(\cdot+t), \beta_{u^{1}(\cdot), d^{1}(\cdot)}\left(u^{1}(\cdot+t), d^{1}(\cdot+t)\right)\right] \\
& =\int_{t}^{\infty} \mathrm{e}^{-\lambda \tau} k\left(y_{x}(\tau), u^{1}(\tau), d^{1}(\tau),\left(\Pi_{1} \delta\right)\left(u^{1}, d^{1}\right)(\tau),\left(\Pi_{2} \delta\right)\left(u^{1}, d^{1}\right)(\tau)\right) \mathrm{d} \tau \\
& \quad-\sum_{\theta_{j}^{1}>t} \mathrm{e}^{-\lambda \theta_{j}^{1}} c^{1}\left(d_{j-1}^{1}, d_{j}^{1}\right) \\
& \quad+\sum_{\left(\Pi_{2} \delta\right)_{1, j}>t} \mathrm{e}^{-\lambda\left(\Pi_{2} \delta\right)_{1, j}} c^{2}\left(\left(\Pi_{2} \delta\right)_{2, j-1},\left(\Pi_{2} \delta\right)_{2, j}\right) \\
& \quad+\sum_{\left(\Pi_{3} \delta\right)_{1, j}>t} \mathrm{e}^{-\lambda\left(\Pi_{3} \delta\right)_{1, j} l\left(\left(\Pi_{3} \delta\right)_{2, j}\right)}
\end{aligned}
$$

Substituting above in the inequality of $V_{-}^{d^{1}(t),\left(\Pi_{2} \bar{\beta}\right)(t)}\left(y_{x}(t)\right)$ and then in the inequality for $W(x)$ will imply

$$
W(x) \geq J_{x}^{d^{1}, d^{2}}\left[u^{1}(\cdot), d^{1}(\cdot), \delta\left(u^{1}, d^{1}\right)(\cdot)\right]-2 \epsilon .
$$

This holds for all $\left(u^{1}(\cdot), d^{1}(\cdot)\right) \in \mathcal{C}^{1, d^{1}}$ and hence $W(x) \geq V_{-}^{d^{1}, d^{2}}(x)-2 \epsilon$. Since $\epsilon>0$ is arbitrary, we get $W(x) \geq V_{-}^{d^{1}, d^{2}}(x)$. 
We now prove the other type of inequality. Fix $\beta \in \Delta^{d^{2}}$ and $\epsilon>0$. Choose $\left(\bar{u}^{1}(\cdot), \bar{d}^{1}(\cdot)\right) \in \mathcal{C}^{1, d^{1}}$ such that

$$
\left.\begin{array}{rl}
W(x) \leq & \int_{0}^{t} \mathrm{e}^{-\lambda s} k\left(y_{x}(s), \bar{u}^{1}(s), \bar{d}^{1}(s), \beta\left(\bar{u}^{1}, \bar{d}^{1}\right)(s)\right) \mathrm{d} s \\
& -\sum_{\theta_{j}^{1}<t} \mathrm{e}^{-\lambda \theta_{j}^{1}} c^{1}\left(d_{j-1}^{1}, d_{j}^{1}\right) \\
& +\sum_{\left(\Pi_{2} \beta\right)_{1, j}<t} \mathrm{e}^{-\lambda\left(\Pi_{2} \beta\right)_{1, j}} c^{2}\left(\left(\Pi_{2} \beta\right)_{2, j-1},\left(\Pi_{2} \beta\right)_{2, j}\right) \\
& +\sum_{\left(\Pi_{3} \beta\right)_{1, j}<t} \mathrm{e}^{-\lambda\left(\Pi_{3} \beta\right)_{1, j}} l\left(\left(\Pi_{3} \beta\right)_{2, j}\right) \\
& +\mathrm{e}^{-\lambda t} V_{-}^{\bar{d}^{1}(t),\left(\Pi_{2} \beta\right)(t)}\left(y_{x}(t)\right)+\epsilon
\end{array}\right\} .
$$

Now for each $u^{1}(\cdot)$, define $\tilde{u}^{1}(\cdot)$ by

$$
\tilde{u}^{1}(s)= \begin{cases}\bar{u}^{1}(s) ; & s \leq t \\ u^{1}(s-t) ; & s>t\end{cases}
$$

Similarly, for each $d^{1}(\cdot)$, we define $\tilde{d}^{1}(\cdot)$. Let

$$
\hat{\beta}\left(u^{1}(\cdot), d^{1}(\cdot)\right)(s)=\beta\left(\tilde{u}^{1}(\cdot), \tilde{d}^{1}(\cdot)\right)(s+t) .
$$

By the definition of $V_{-}$, we can choose $\left(u^{1}(\cdot), d^{1}(\cdot)\right) \in \mathcal{C}^{1, \bar{d}^{1}(t)}$ such that

$$
\begin{aligned}
& V_{-}^{\bar{d}^{1}(t),\left(\Pi_{2} \beta\right)(t)}\left(y_{x}(t)\right) \\
& \quad \leq J_{y_{x}(t)}^{\bar{d}^{1}(t),\left(\Pi_{2} \beta\right)(t)}\left[u^{1}(\cdot+t), d^{1}(\cdot+t), \hat{\beta}\left(u^{1}, d^{1}\right)(\cdot+t)\right]+\epsilon \mathrm{e}^{\lambda t} .
\end{aligned}
$$

Now, combining (2.2) and (2.3), we get

$$
\begin{aligned}
W(x) \leq & \int_{0}^{t} \mathrm{e}^{-\lambda s} k\left(y_{x}(s), \bar{u}^{1}(s), \bar{d}^{1}(s), \beta\left(\bar{u}^{1}, \bar{d}^{1}\right)(s)\right) \mathrm{d} s-\sum_{\theta_{j}^{1}<t} \mathrm{e}^{-\lambda \theta_{j}^{1}} c^{1}\left(d_{j-1}^{1}, d_{j}^{1}\right) \\
& +\sum_{\left(\Pi_{2} \beta\right)_{1, j}<t} \mathrm{e}^{-\lambda \Pi_{2} \beta_{1, j}} c^{2}\left(\left(\Pi_{2} \beta\right)_{2, j-1},\left(\Pi_{2} \beta\right)_{2, j}\right) \\
& +\sum_{\Pi_{3} \beta_{1, j}<t} \mathrm{e}^{-\lambda\left(\Pi_{3} \beta\right)_{1, j}} l\left(\left(\Pi_{3} \beta\right)_{2, j}\right) \\
& +\mathrm{e}^{-\lambda t} J_{y_{x}(t)}^{\bar{d}^{1}(t),\left(\Pi_{2} \beta\right)(t)}\left[u^{1}(\cdot), d^{1}(\cdot), \hat{\beta}\left(u^{1}(\cdot), d^{1}(\cdot)\right)\right]+2 \epsilon .
\end{aligned}
$$

By change of variables, it follows that

$$
W(x) \leq J_{x}^{d^{1}, d^{2}}\left[\tilde{u}^{1}(\cdot), \tilde{d}^{1}(\cdot), \beta\left(\tilde{u}^{1}, \tilde{d}^{1}\right)(\cdot)\right]+2 \epsilon .
$$

This holds for any $\beta \in \Delta^{d^{2}}$ and hence

$$
W(x) \leq V_{-}^{d^{1}, d^{2}}(x)+2 \epsilon .
$$

The proof is now complete, since $\epsilon$ is arbitrary. 
Proof of Lemma 2.3. We first prove (i) and (ii). By the definition of $V^{-}$, for any $\bar{d}^{2} \neq d^{2}$,

$$
V_{-}^{d^{1}, d^{2}}(x) \leq V_{-}^{d^{1}, \bar{d}^{2}}(x)+c^{2}\left(d^{2}, \bar{d}^{2}\right) .
$$

From this we get

$$
V_{-}^{d^{1}, d^{2}}(x) \leq M_{-}^{d^{1}, d^{2}}\left[V_{-}\right](x) .
$$

The inequality

$$
V_{-}^{d^{1}, d^{2}}(x) \geq M_{+}^{d^{1}, d^{2}}\left[V_{-}\right](x)
$$

can be proved in a similar fashion.

Clearly for any $\xi \in K$ and $\beta \in \Delta^{d^{2}}$,

$$
V_{-}^{d^{1}, d^{2}}(x) \leq \sup _{\mathcal{C}^{1, d^{1}}} J_{x+\xi}^{d^{1}, d^{2}}\left[u^{1}(\cdot), d^{1}(\cdot), \beta\left(u^{1}, d^{1}\right)(\cdot)\right]+l(\xi) .
$$

Taking infimum over $\beta \in \Delta^{d^{2}}$ and then over $\xi \in K$, we obtain

$$
V_{-}^{d^{1}, d^{2}}(x) \leq N\left[V_{-}^{d^{1}, d^{2}}\right](x) .
$$

We now turn to the proof of (iii). By Lemma 2.1, for each $t \geq 0$, there exists $\left(u^{1, t}(\cdot), d^{1, t}(\cdot)\right) \in \mathcal{C}^{1, d^{1}}$ such that

$$
\begin{aligned}
V_{-}^{d^{1}, d^{2}}(x)-t^{2} \leq & \int_{0}^{t} \mathrm{e}^{-\lambda s} k\left(y_{x}(s), u^{1, t}(s), d^{1, t}(s), \bar{\beta}\left(u^{1, t}, d^{1, t}\right)(s)\right) \mathrm{d} s \\
& -\sum_{\theta_{j}^{1, t}<t} \mathrm{e}^{-\lambda \theta_{j}^{1, t}} c^{1}\left(d_{j-1}^{1, t}, d_{j}^{1, t}\right)+\mathrm{e}^{-\lambda t} V_{-}^{d^{1, t}(t),\left(\Pi_{2} \bar{\beta}\right)(t)}\left(y_{x}(t)\right) .
\end{aligned}
$$

It is enough to show that, for some $t_{0}>0, \theta_{1}^{1, t} \geq t$ for all $0 \leq t \leq t_{0}$. If this does not happen, then there would exist a sequence $t_{n} \downarrow 0$ such that $\theta_{1}^{1, t_{n}}<t_{n}$ for all $n$. This would imply that

$$
\begin{aligned}
V_{-}^{d^{1}, d^{2}}(x)-\left(\theta_{1}^{1, t_{n}}\right)^{2} \leq & \int_{0}^{\theta_{1}^{1, t_{n}}} \mathrm{e}^{-\lambda s} k\left(y_{x}(s), u^{1, t_{n}}(s), d^{1, t_{n}}(s), \bar{\beta}\left(u^{1, t_{n}}, d^{1, t_{n}}\right)(s)\right) \mathrm{d} s \\
& -\mathrm{e}^{-\lambda \theta_{1}^{1, t_{n}}} c^{1}\left(d^{1}, d_{1}^{1, t_{n}}\right)+\mathrm{e}^{-\lambda \theta_{1}^{1, t_{n}}} V_{-}^{d_{1}^{1, t_{n}}, d^{2}}\left(y_{x}\left(\theta_{1}^{1, t_{n}}\right)\right) .
\end{aligned}
$$

We may assume that for all $n, d_{1}^{1, t_{n}}=\bar{d}^{1} \neq d^{1}$. Now by letting $n \rightarrow \infty$ in the above inequality, we get

$$
\begin{aligned}
V_{-}^{d^{1}, d^{2}}(x) & \leq-c^{1}\left(d^{1}, \bar{d}^{1}\right)+V_{-}^{\bar{d}^{1}, d^{2}}(x) \\
& \leq M_{+}^{d^{1}, d^{2}}\left[V_{-}\right](x) .
\end{aligned}
$$

This contradicts the hypothesis that strict inequality holds in (i) and the proof of (iii) is now complete. 
We next prove (iv). By Lemma 2.1, for each $t>0$, there exists $\beta^{t} \in \Delta^{d^{2}}$ such that

$$
\begin{aligned}
V_{-}^{d^{1}, d^{2}}(x)+t^{2} \geq & \int_{0}^{t} \mathrm{e}^{-\lambda s} k\left(y_{x}(s), \bar{u}^{1}, d^{1}, \beta^{t}\left(\bar{u}^{1}, d^{1}\right)(s)\right) \mathrm{d} s+\mathrm{e}^{-\lambda t} V_{-}^{d^{1},\left(\Pi_{2} \beta^{t}\right)(t)}\left(y_{x}(t)\right) \\
& +\sum_{\left(\Pi_{2} \beta^{t}\right)_{1, j}<t} \mathrm{e}^{-\lambda \theta_{j}^{2, t}} c^{2}\left(d_{j-1}^{2, t}, d_{j}^{2, t}\right) \\
& +\sum_{\left(\Pi_{3} \beta^{t}\right)_{1, j}<t} \mathrm{e}^{-\lambda \tau_{j}^{t} l\left(\xi_{j}^{t}\right)}
\end{aligned}
$$

It is enough to show that, for some $t_{0}>0, \min \left(\theta_{1}^{2, t}, \tau_{1}^{t}\right) \geq t$ for all $0 \leq t \leq t_{0}$. If this were not true, then (without any loss of generality) there would be a sequence $t_{n} \downarrow 0$ and two cases to consider. In the first case, $\theta_{1}^{2, t_{n}} \leq \min \left(t_{n}, \tau_{1}^{t_{n}}\right)$ whereas in the second case $\tau_{1}^{t_{n}} \leq \min \left(t_{n}, \theta_{1}^{2, t_{n}}\right)$. By dropping to a subsequence if necessary and proceeding as in the proof of (iii), we get $V_{-}^{d^{1}, d^{2}}(x) \geq M_{-}^{d^{1}, d^{2}}\left[V_{-}\right](x)$ in case 1 and $V_{-}^{d^{1}, d^{2}}(x) \geq N\left[V_{-}^{d^{1}, d^{2}}\right](x)$ in case 2 respectively. This contradicts our hypothesis that strict inequality holds in (ii) and the proof is complete.

Remark 2.5. From the proofs it is clear that, instead of the term $t^{2}$ in the statement of Lemma 2.3 and Lemma 2.4, we can take any modulus $\rho(t)$.

We now state the following result (Corollary 4.9, [4]) which is useful in proving that $V_{-}\left(\right.$resp. $\left.V_{+}\right)$is a viscosity solution of $(\mathrm{HJI}+)($ resp. $(\mathrm{HJI}-))$.

Lemma 2.6. Let $\Phi \in \mathcal{T}, \hat{x} \in \mathbb{E}$ and $D_{A}^{-} \Phi(\hat{x})<\infty$. If $v(\cdot) \in L^{1}([0, T] ; \mathbb{E})$ and $y(\cdot)$ solves

$$
\begin{aligned}
& \dot{y}(t)+A y(t)=v(t) ; \quad 0 \leq t \leq T, \\
& y(0)=\hat{x},
\end{aligned}
$$

then as $t \rightarrow 0$,

$$
\begin{aligned}
\mathrm{e}^{-\lambda t} \Phi(y(t))-\Phi(\hat{x}) \leq & \frac{\mathrm{e}^{-\lambda t}-1}{\lambda} D_{A}^{-} \Phi(\hat{x})+L(\psi) \int_{0}^{t} \mathrm{e}^{-\lambda s}\|v(s)\| \mathrm{d} s \\
& +\int_{0}^{t} \mathrm{e}^{-\lambda s}[\langle D \phi(y(s)), v(s)\rangle-\lambda \Phi(y(s))] \mathrm{d} s+o(t),
\end{aligned}
$$

uniformly for all $v(\cdot)$ uniformly integrable on $(0, T)$.

We are now ready to prove that $V_{-}$(resp. $\left.V_{+}\right)$is an approximate viscosity solution of $(\mathrm{HJI}+)($ resp. $(\mathrm{HJI}-))$.

Theorem 2.7. The lower value function $V_{-}$is an approximate viscosity solution of $(\mathrm{HJ}+)$ and the upper value function $V_{+}$is an approximate viscosity solution of (HJI-).

Proof. We prove that $V_{-}$is an approximate viscosity solution of (HJI+). The other part can be proved in an analogous manner.

Let $C_{R}$ be such that $\left\|f\left(y_{x}(t), u^{1}(t), d^{1}(t), u^{2}(t), d^{2}(t), \xi(t)\right)\right\| \leq C_{R}$ for all $0 \leq t \leq 1$, $\|x\| \leq R,\left(u^{1}(\cdot), d^{1}(\cdot)\right) \in \mathcal{C}^{1}$ and $\left(u^{2}(\cdot), d^{2}(\cdot), \xi(\cdot)\right) \in \mathcal{C}^{2}$. 
We first prove that $V_{-}$is an approximate subsolution of (HJI1+). Let $\left(\hat{x}, d^{1}, d^{2}\right) \in$ $\mathbb{E} \times D^{1} \times D^{2}$ and $\Phi=\phi+\psi \in \mathcal{T}$ be such that $V_{-}^{d^{1}, d^{2}}-\Phi$ has a local maximum at $\hat{x} \in B_{R}(0)$. Without any loss of generality, we may assume that $V_{-}^{d^{1}, d^{2}}(\hat{x})=\Phi(\hat{x})$. If $V_{-}^{d^{1}, d^{2}}(\hat{x})=M_{+}^{d^{1}, d^{2}}\left[V_{-}\right](\hat{x})$, then we are done. Assume that $V_{-}^{d^{1}, d^{2}}(\hat{x})>M_{+}^{d^{1}, d^{2}}\left[V_{-}\right](\hat{x})$. It suffices to show that

$$
\lambda \Phi(\hat{x})+D_{A}^{-} \Phi(\hat{x})+H_{+}^{d^{1}, d^{2}}(\hat{x}, D \phi(\hat{x}))-C_{R} L(\psi)=: r \leq 0 .
$$

If possible, let $r>0$. This implies that for every $u^{1} \in U^{1}$, there exists $u^{2}=u^{2}\left(u^{1}\right)$ such that

$$
\begin{aligned}
& \lambda \Phi(\hat{x})+D_{A}^{-} \Phi(\hat{x})-\left\langle D \phi(\hat{x}), f\left(\hat{x}, u^{1}, d^{1}, u^{2}, d^{2}\right)\right\rangle \\
& -k\left(\hat{x}, u^{1}, d^{1}, u^{2}, d^{2}\right)-C_{R} L(\psi) \geq \frac{r}{2} .
\end{aligned}
$$

By Proposition 3.2 of [4], for $t$ small enough, there exists $\beta^{t} \in \Delta_{0}^{d^{2}}$ such that

$$
\begin{gathered}
\lambda \Phi(\hat{x})+D_{A}^{-} \Phi(\hat{x})-\left\langle D \phi(\hat{x}), f\left(\hat{x}, u^{1}(s), d^{1}, \beta^{t}\left(u^{1}(\cdot), d^{1}\right)(s)\right)\right\rangle \\
-k\left(\hat{x}, u^{1}(s), d^{1}, \beta^{t}\left(u^{1}(\cdot), d^{1}\right)(s)\right)-C_{R} L(\psi) \geq \frac{r}{2}
\end{gathered}
$$

for all $u^{1}(\cdot) \in \mathcal{U}^{1}[0, t]$ and a.e. $s \in[0, t]$.

This yields

$$
\begin{aligned}
& \lambda \Phi\left(y_{\hat{x}}(s)\right)+D_{A}^{-} \Phi(\hat{x})-\left\langle D \phi\left(y_{\hat{x}}(s)\right), f\left(y_{\hat{x}}(s), u^{1}(s), d^{1}, \beta^{t}\left(u^{1}(\cdot), d^{1}\right)(s)\right)\right\rangle \\
& \quad-k\left(y_{\hat{x}}(s), u^{1}(s), d^{1}, \beta^{t}\left(u^{1}(\cdot), d^{1}\right)(s)\right)-C_{R} L(\psi) \geq \frac{r}{2}
\end{aligned}
$$

for a.e. $s \in[0, t]$ and $t$ small enough.

Multiplying throughout by $\mathrm{e}^{-\lambda s}$ and integrating from $s=0$ to $s=t$, we get

$$
\left.\begin{array}{l}
{\left[D_{A}^{-} \Phi(\hat{x})-C_{R} L(\psi)-\frac{r}{2}\right]\left[\frac{\mathrm{e}^{-\lambda t}-1}{-\lambda}\right]} \\
\quad-\int_{0}^{t} \mathrm{e}^{-\lambda s} k\left(y_{\hat{x}}(s), u^{1}(s), d^{1}, \beta^{t}\left(u^{1}(\cdot), d^{1}\right)(s)\right) \mathrm{d} s \\
\quad+\int_{0}^{t} \mathrm{e}^{-\lambda s}\left[\lambda \Phi\left(y_{\hat{x}}(s)\right)\right. \\
\left.\quad-\left\langle D \phi\left(y_{\hat{x}}(s)\right), f\left(y_{\hat{x}}(s), u^{1}(s), d^{1}, \beta^{t}\left(u^{1}(\cdot), d^{1}\right)(s)\right)\right\rangle\right] \mathrm{d} s \geq 0
\end{array}\right\}
$$

By Lemma 2.3(iii), for $t$ small enough, there exists $u^{1, t}(\cdot) \in \mathcal{U}^{1}[0, t]$ such that

$$
\begin{aligned}
V_{-}^{d^{1}, d^{2}}(\hat{x})-o(t) \leq & \int_{0}^{t} \mathrm{e}^{-\lambda s} k\left(y_{\hat{x}}(s), u^{1, t}(s), d^{1}, \beta^{t}\left(u^{1, t}(\cdot), d^{1}\right)(s)\right) \mathrm{d} s \\
& +\mathrm{e}^{-\lambda t} V_{-}^{d^{1}, d^{2}}\left(y_{\hat{x}}(t)\right) .
\end{aligned}
$$


We now claim that $D_{A}^{-} \Phi(\hat{x})<\infty$. We may take $\Phi$ to be Lipschitz.

$$
\begin{aligned}
\left\|\Phi(\hat{x})-\Phi\left(y_{\hat{x}}(\delta)\right)\right\| & \leq\left\|\Phi(\hat{x})-\mathrm{e}^{-\lambda \delta} \Phi\left(y_{\hat{x}}(\delta)\right)\right\|+\left(\mathrm{e}^{-\lambda \delta}-1\right)\left\|\Phi\left(y_{\hat{x}}(\delta)\right)\right\| \\
& \leq o(\delta)+C_{\hat{x}} \delta+C_{\hat{x}}\left(\mathrm{e}^{-\lambda \delta}-1\right) .
\end{aligned}
$$

Therefore

$$
\begin{aligned}
\|\Phi(\hat{x})-\Phi(S(\delta) \hat{x})\| & \leq\left\|\Phi(\hat{x})-\Phi\left(y_{\hat{x}}(\delta)\right)\right\|+L(\Phi) C_{\hat{x}} \delta \\
& \leq o(\delta)+C \delta+C\left(\mathrm{e}^{-\lambda \delta}-1\right) .
\end{aligned}
$$

This proves the claim that $D_{A}^{-} \Phi(\hat{x})<\infty$ and hence from Lemma 2.6, it follows that as $t \rightarrow 0$,

$$
\left.\begin{array}{rl}
\mathrm{e}^{-\lambda t} \Phi\left(y_{\hat{x}}(t)\right)-\Phi(\hat{x}) \leq & \frac{\mathrm{e}^{-\lambda t}-1}{\lambda} D_{A}^{-} \Phi(\hat{x}) \\
& +L(\psi) \int_{0}^{t} \mathrm{e}^{-\lambda s}\left\|f\left(y_{\hat{x}}(s), u^{1, t}(s), d^{1}, \beta^{t}\left(u^{1, t}(\cdot), d^{1}\right)(s)\right)\right\| \mathrm{d} s \\
& +\int_{0}^{t} \mathrm{e}^{-\lambda s}\left[\left\langleD \phi\left(y_{\hat{x}}(s)\right), f\left(y_{\hat{x}}(s), u^{1, t}(s), d^{1},\right.\right.\right. \\
& \left.\left.\left.\beta^{t}\left(u^{1, t}(\cdot), d^{1}\right)(s)\right)\right\rangle-\lambda \Phi\left(y_{\hat{x}}(s)\right)\right] \mathrm{d} s+o(t) .
\end{array}\right\}
$$

Combining (2.4), (2.5) and (2.6), we obtain

$$
\begin{aligned}
{\left[\frac{\mathrm{e}^{-\lambda t}-1}{\lambda}\right] \frac{r}{2} \geq } & \mathrm{e}^{-\lambda t} \Phi\left(y_{\hat{x}}(t)\right)-\Phi(\hat{x}) \\
& +\int_{0}^{t} \mathrm{e}^{-\lambda s} k\left(y_{\hat{x}}(s), u^{1, t}(s), d^{1}, \beta^{t}\left(u^{1, t}(\cdot), d^{1}\right)(s)\right) \mathrm{d} s+o(t) \\
\geq & o(t) .
\end{aligned}
$$

This contradiction proves that $V_{-}$is an approximate subsolution of (HJI1+).

To prove that $V_{-}$is an approximate supersolution of $(\mathrm{HJI} 1+)$, let $\hat{x} \in B_{R}(0)$ be a local minimum of $V_{-}^{d^{1}, d^{2}}-\Phi$. Without any loss of generality, we may assume that $V_{-}^{d^{1}, d^{2}}(\hat{x})=$ $\Phi(\hat{x})$. If $V_{-}^{d^{1}, d^{2}}(\hat{x})=M_{-}^{d^{1}, d^{2}}\left[V_{-}\right](\hat{x})$ or $V_{-}^{d^{1}, d^{2}}(\hat{x})=N\left[V_{-}^{d^{1}, d^{2}}\right](\hat{x})$, then we are done. Assume that $V_{-}^{d^{1}, d^{2}}(\hat{x})<\min \left(M_{-}^{d^{1}, d^{2}}\left[V_{-}\right](\hat{x}), N\left[V_{-}^{d^{1}, d^{2}}\right](\hat{x})\right)$. In this case, we need to show that

$$
\lambda \Phi(x)+D_{A}^{+} \Phi(\hat{x})+H_{+}^{d^{1}, d^{2}}(\hat{x}, D \phi(\hat{x}))+C_{R} L(\psi)=: \hat{r} \geq 0 .
$$

If possible, let $\hat{r}<0$. Then

$$
\begin{gathered}
\lambda \Phi(\hat{x})+D_{A}^{+} \Phi(\hat{x})-\left\langle D \phi(\hat{x}), f\left(\hat{x}, \bar{u}^{1}, d^{1}, u^{2}, d^{2}\right)\right\rangle \\
-k\left(\left(\hat{x}, \bar{u}^{1}, d^{1}, u^{2}, d^{2}\right)+C_{R} L(\psi) \leq \frac{\hat{r}}{2},\right.
\end{gathered}
$$


for some $\bar{u}^{1} \in U^{1}$ and all $u^{2} \in U^{2}$. This implies that, for all $\beta \in \Delta_{0}^{d^{2}}$ and $s$ small enough

$$
\begin{aligned}
& \lambda \Phi\left(y_{\hat{x}}(s)\right)+D_{A}^{+} \Phi(\hat{x})-\left\langle D \phi(y(s)), f\left(y_{\hat{x}}(s), \bar{u}^{1}, d^{1}, \beta\left(\bar{u}^{1}, d^{1}\right)(s)\right)\right\rangle \\
& -k\left(y_{\hat{x}}(s), \bar{u}^{1}, d^{1}, \beta\left(\bar{u}^{1}, d^{1}\right)(s)\right)+C_{R} L(\psi) \leq \frac{\hat{r}}{4} .
\end{aligned}
$$

Multiplying throughout by $\mathrm{e}^{-\lambda s}$ and integrating from 0 to $t$, we get

$$
\left.\begin{array}{l}
\left\{D_{A}^{+} \Phi(\hat{x})+C_{R} L(\psi)-\frac{\hat{r}}{4}\right\}\left[\frac{\mathrm{e}^{-\lambda t}-1}{-\lambda}\right] \\
\quad+\int_{0}^{t} \mathrm{e}^{-\lambda s}\left[\lambda \Phi\left(y_{\hat{x}}(s)\right)-\left\langle D \phi(y(s)), f\left(y_{\hat{x}}(s), \bar{u}^{1}, d^{1}, \beta\left(\bar{u}^{1}, d^{1}\right)(s)\right)\right\rangle\right] \mathrm{d} s \\
\quad-\int_{0}^{t} \mathrm{e}^{-\lambda s} k\left(y_{\hat{x}}(s), \bar{u}^{1}, d^{1}, \beta\left(\bar{u}^{1}, d^{1}\right)(s)\right) \mathrm{d} s \leq 0
\end{array}\right\} .
$$

Now by Lemma 2.3(iv), for $t$ small enough, there exists $\beta^{t} \in \Delta_{0}^{d^{2}}$ such that

$$
\begin{aligned}
& V_{-}^{d^{1}, d^{2}}(\hat{x})+o(t) \\
& \quad \geq \int_{0}^{t} \mathrm{e}^{-\lambda s} k\left(y_{\hat{x}}(s), \bar{u}^{1}, d^{1}, \beta^{t}\left(\bar{u}^{1}, d^{1}\right)(s)\right) \mathrm{d} s+\mathrm{e}^{-\lambda t} V_{-}^{d^{1}, d^{2}}\left(y_{\hat{x}}(t)\right) .
\end{aligned}
$$

Now proceeding as in the first part of the theorem, we can show that $D_{A}^{+} \Phi(\hat{x})>-\infty$. Using Lemma 2.6 for $-\Phi$, we get

$$
\begin{aligned}
-\mathrm{e}^{-\lambda t} \Phi\left(y_{\hat{x}}(t)\right)+\Phi(\hat{x}) \leq & \frac{\mathrm{e}^{-\lambda t}-1}{-\lambda} D_{A}^{+} \Phi(\hat{x}) \\
& +L(\psi) \int_{0}^{t} \mathrm{e}^{-\lambda s}\left\|f\left(y_{\hat{x}}(s), u^{1, t}(s), d^{1}, \beta^{t}\left(u^{1, t}(\cdot), d^{1}\right)(s)\right)\right\| \mathrm{d} s \\
& -\int_{0}^{t} \mathrm{e}^{-\lambda s}\left[\left\langleD \phi\left(y_{\hat{x}}(s)\right), f\left(y_{\hat{x}}(s), u^{1, t}(s), d^{1},\right.\right.\right. \\
& \left.\left.\left.\beta^{t}\left(u^{1, t}(\cdot), d^{1}\right)(s)\right)\right\rangle-\lambda \Phi\left(y_{\hat{x}}(s)\right)\right] \mathrm{d} s+o(t) .
\end{aligned}
$$

From (2.7), (2.8) and (2.9), we get

$$
\frac{\hat{r}}{4}\left[\frac{\mathrm{e}^{-\lambda t}-1}{\lambda}\right] \leq o(t) .
$$

This is a contradiction and proves the fact that $V_{-}$is an approximate supersolution of $(\mathrm{HJI} 1+)$.

In a similar fashion, we can show that $V_{-}$is an approximate viscosity solution of (HJI2+). Hence $V_{-}$is an approximate viscosity solution of (HJI+). 


\section{Existence of value}

In this section we first prove the uniqueness of solutions of (HJI+) and (HJI-). Next we prove that uniqueness result for (HJI+) and (HJI-) holds true even if one is a viscosity solution and other is an approximate viscosity solution. Finally under Isaacs' type minmax condition we show that game has a value proving the main theorem of the paper. Now we state and prove two lemmas needed in the proof of uniqueness of (HJI+) and (HJI-).

Lemma 3.1. Assume (A2). Let $w$ be uniformly continuous. If

$$
w^{d^{1}, d^{2}}\left(y_{0}\right)=N\left[w^{d^{1}, d^{2}}\right]\left(y_{0}\right)=w^{d^{1}, d^{2}}\left(y_{0}+\xi_{0}\right)+l\left(\xi_{0}\right),
$$

then there exists $\sigma>0$ (which depends only on $w$ ) such that for all $y \in \bar{B}\left(y_{0}+\xi_{0}, \sigma\right)$,

$$
w^{d^{1}, d^{2}}(y)<N\left[w^{d^{1}, d^{2}}\right](y) .
$$

Proof. The proof closely mimics the corresponding result in the finite dimensional case [8]. We however prove it for the sake of completeness. Let

$$
w^{d^{1}, d^{2}}\left(y_{0}\right)=N\left[w^{d^{1}, d^{2}}\right]\left(y_{0}\right)=w^{d^{1}, d^{2}}\left(y_{0}+\xi_{0}\right)+l\left(\xi_{0}\right) .
$$

Then, for every $\xi_{1} \in K$,

$$
\begin{aligned}
w^{d^{1}, d^{2}}\left(y_{0}+\xi_{0}+\xi_{1}\right)+l\left(\xi_{1}\right)-w^{d^{1}, d^{2}}\left(y_{0}+\xi_{0}\right)= & w^{d^{1}, d^{2}}\left(y_{0}+\xi_{0}+\xi_{1}\right)+l\left(\xi_{1}\right) \\
& -w^{d^{1}, d^{2}}\left(y_{0}\right)+l\left(\xi_{0}\right) \\
\geq & -l\left(\xi_{0}+\xi_{1}\right)+l\left(\xi_{0}\right)+l\left(\xi_{1}\right) .
\end{aligned}
$$

Now,

$$
N\left[w^{d^{1}, d^{2}}\right]\left(y_{0}+\xi_{0}\right)=\inf _{\xi_{1} \in K} w^{d^{1}, d^{2}}\left(y_{0}+\xi_{0}+\xi_{1}\right)+l\left(\xi_{1}\right) .
$$

By (1.3), this infimum will be attained in some $R$ ball. Hence we can write

$$
N\left[w^{d^{1}, d^{2}}\right]\left(y_{0}+\xi_{0}\right)=\inf _{\xi_{1} \in K,\left|\xi_{1}\right|<R} w^{d^{1}, d^{2}}\left(y_{0}+\xi_{0}+\xi_{1}\right)+l\left(\xi_{1}\right) .
$$

Now, taking infimum over $\left|\xi_{1}\right|<R$ on both sides of the earlier inequality we will have

$$
\begin{aligned}
N\left[w^{d^{1}, d^{2}}\left(y_{0}+\xi_{0}\right)\right]-w^{d^{1}, d^{2}}\left(y_{0}+\xi_{0}\right) & \geq \inf _{\xi_{1} \in K,\left|\xi_{1}\right|<R}\left[l\left(\xi_{0}\right)+l\left(\xi_{1}\right)-l\left(\xi_{0}+\xi_{1}\right)\right] \\
& =\bar{l}>0 .
\end{aligned}
$$

By using uniform continuity of $w^{d^{1}, d^{2}}$ and $N\left[w^{d^{1}, d^{2}}\right]$ we get a $\sigma$ such that for all $y \in$ $\bar{B}\left(y_{0}+\xi_{0}, \sigma\right)$,

$$
w^{d^{1}, d^{2}}(y)<N\left[w^{d^{1}, d^{2}}\right](y) .
$$


Lemma 3.2. Assume (A1) and (A2). Then the following results are true.

(i) Any supersolution $w$ of $(\mathrm{HJI} 1+)$ satisfies $w^{d^{1}, d^{2}} \geq M_{+}^{d^{1}, d^{2}}[w]$ for all $d^{1}, d^{2}$.

(ii) Any subsolution $w$ of (HJI2+) satisfies $w^{d^{1}, d^{2}} \leq \min \left(M_{-}^{d^{1}, d^{2}}[w], N\left[w^{d^{1}}, d^{2}\right]\right)$ for all $d^{1}, d^{2}$.

Proof. Let $w$ be a supersolution of (HJI1+). If possible, let

$$
w^{d^{1}, d^{2}}\left(x_{0}\right)<M_{+}^{d^{1}, d^{2}}[w]\left(x_{0}\right)
$$

By continuity, the above holds for all $x$ in an open ball $B$ around $x_{0}$. As in Lemma 1.8(d), p. 30 in [1], we can show that there exists $y_{0} \in B$ and a smooth map $\phi$ such that $w^{d^{1}, d^{2}}-\phi$ has local minimum at $y_{0}$. Since $w$ is a supersolution of (HJI1+), this will lead to

$$
w^{d^{1}, d^{2}}\left(y_{0}\right) \geq M_{+}^{d^{1}, d^{2}}[w]\left(y_{0}\right),
$$

a contradiction. This proves (i). The proof of (ii) is similar.

Next we present the proof of the uniqueness theorem.

Theorem 3.3. Assume (A1) and (A2). Let $v$ and $w \in \mathrm{BUC}\left(\mathbb{E} ; \mathbb{R}^{m_{1} \times m_{2}}\right)$ be viscosity solutions of $(\mathrm{HJI}+)($ or $(\mathrm{HJI}-))$. Then $v=w$.

Proof. We prove the uniqueness for (HJI+). The result for $(\mathrm{HJI}-)$ is similar.

Let $v$ and $w$ be viscosity solutions of (HJI+). We prove $v^{d^{1}, d^{2}} \leq w^{d^{1}, d^{2}}$ for all $d^{1}, d^{2}$. In a similar fashion we can prove that $w^{d^{1}, d^{2}} \leq v^{d^{1}, d^{2}}$ for all $d^{1}, d^{2}$.

For $\left(d^{1}, d^{2}\right) \in D^{1} \times D^{2}$, define $\Phi^{d^{1}, d^{2}}: \mathbb{E} \times \mathbb{E} \rightarrow \mathbb{R}$ by

$$
\Phi^{d^{1}, d^{2}}(x, y)=v^{d^{1}, d^{2}}(x)-w^{d^{1}, d^{2}}(y)-\frac{|x-y|^{2}}{2 \epsilon}-\kappa\left[\langle x\rangle^{\bar{m}}+\langle y\rangle^{\bar{m}}\right],
$$

where $\bar{m} \in(0,1) \cap\left(0, \frac{\lambda}{\|f\|_{\infty}}\right)$ is fixed, $\kappa, \epsilon \in(0,1)$ are parameters, and $\langle x\rangle^{\bar{m}}=(1+$ $\left.\|x\|^{2}\right)^{\bar{m} / 2}$.

Note that $x \rightarrow\langle x\rangle^{\bar{m}}$ is Lipschitz continuous with Lipschitz constant 1 .

We first fix $\kappa>0$. Let $\left(x_{\epsilon}, y_{\epsilon}\right)$ and $\left(d_{\epsilon}^{1}, d_{\epsilon}^{2}\right)$ be such that

$$
\Phi^{d_{\epsilon}^{1}, d_{\epsilon}^{2}}\left(x_{\epsilon}, y_{\epsilon}\right)=\sup _{x, y} \max _{d^{1}, d^{2}} \Phi^{d^{1}, d^{2}}(x, y)-\epsilon\left[d\left(x, x_{\epsilon}\right)+d\left(y, y_{\epsilon}\right)\right]
$$

and

$$
\Phi^{d_{\epsilon}^{1}, d_{\epsilon}^{2}}\left(x_{\epsilon}, y_{\epsilon}\right) \geq \sup _{x, y} \max _{d^{1}, d^{2}} \Phi^{d^{1}, d^{2}}(x, y)-\epsilon
$$

Here $d(\cdot, \cdot)$ is the Tataru's distance defined by

$$
d(x, y)=\inf _{t \geq 0}[t+\|x-S(t) y\|]
$$


(see [2] for more details). By Lemma 3.2, we have

$$
\begin{gathered}
w^{d_{\epsilon}^{1}, d_{\epsilon}^{2}}\left(y_{\epsilon}\right) \leq N\left[w^{d_{\epsilon}^{1}, d_{\epsilon}^{2}}\right]\left(y_{\epsilon}\right), \\
w^{d_{\epsilon}^{1}, d_{\epsilon}^{2}}\left(y_{\epsilon}\right) \leq M_{-} d_{\epsilon}^{1}, d_{\epsilon}^{2}[w]\left(y_{\epsilon}\right), \\
v^{d_{\epsilon}^{1}, d_{\epsilon}^{2}}\left(x_{\epsilon}\right) \geq M_{+} d_{\epsilon}^{1}, d_{\epsilon}^{2}[v]\left(x_{\epsilon}\right) .
\end{gathered}
$$

If we have strict inequality in all the above three inequalities (that is, (3.1), (3.2) and (3.3)), then by the definition of viscosity sub and super solutions we will have

$$
\begin{aligned}
& \lambda v^{d_{\epsilon}^{1}, d_{\epsilon}^{2}}\left(x_{\epsilon}\right)+D_{A}^{-} \Phi_{1}\left(x_{\epsilon}\right)+H_{+, \underline{\epsilon}}^{d_{\epsilon}^{1}, d_{\epsilon}^{2}}\left(x_{\epsilon}, \frac{x_{\epsilon}-y_{\epsilon}}{\epsilon}+\kappa \bar{m}\left\langle x_{\epsilon}\right\rangle^{\bar{m}-2} x_{\epsilon}\right) \leq 0, \\
& \lambda w^{d_{\epsilon}^{1}, d_{\epsilon}^{2}}\left(y_{\epsilon}\right)+D_{A}^{+} \Phi_{2}\left(y_{\epsilon}\right)+H_{+, \bar{\epsilon}}^{d_{\epsilon}^{1}, d_{\epsilon}^{2}}\left(y_{\epsilon}, \frac{x_{\epsilon}-y_{\epsilon}}{\epsilon}-\kappa \bar{m}\left\langle y_{\epsilon}\right\rangle^{\bar{m}-2} y_{\epsilon}\right) \geq 0,
\end{aligned}
$$

where

$$
\begin{aligned}
\Phi_{1}(x) & =\frac{\left|x-y_{\epsilon}\right|^{2}}{2 \epsilon}+\kappa\langle x\rangle^{\bar{m}}+\epsilon d\left(x, x_{\epsilon}\right), \\
-\Phi_{2}(y) & =\frac{\left|x_{\epsilon}-y\right|^{2}}{2 \epsilon}+\kappa\langle y\rangle^{\bar{m}}+\epsilon d\left(y, y_{\epsilon}\right) .
\end{aligned}
$$

In this case we can proceed by the usual comparison principle method as in [2].

Therefore it is enough to show that for a proper auxiliary function strict inequality occurs in (3.1), (3.2) and (3.3) at the maximizer. We achieve this in the following three steps.

Step 1 . There are two cases to consider; either there is no sequence $\epsilon_{n} \downarrow 0$ such that strict inequality holds in (3.1) or there is some sequence for which strict inequality holds in (3.2).

If there is no sequence $\epsilon_{n} \downarrow 0$ such that

$$
w^{d_{\epsilon_{n}}^{1}, d_{\epsilon_{n}}^{2}}\left(y_{\epsilon_{n}}\right)<N\left[w^{d_{\epsilon_{n}}^{1}, d_{\epsilon_{n}}^{2}}\right]\left(y_{\epsilon_{n}}\right) \text { for all } n,
$$

then we have equality in (3.1) for all $\epsilon$ in some interval $\left(0, \epsilon_{0}\right)$. By the definition of $N$ and the assumptions (A2), for each $\epsilon \in\left(0, \epsilon_{0}\right)$, there exists $\xi_{\epsilon} \in K$ such that

$$
M_{0}:=\sup _{0<\epsilon<\epsilon_{0}}\left\|\xi_{\epsilon}\right\|<\infty
$$

and

$$
w^{d_{\epsilon}^{1}, d_{\epsilon}^{2}}\left(y_{\epsilon}\right)=N\left[w^{d_{\epsilon}^{1}, d_{\epsilon}^{2}}\right]\left(y_{\epsilon}\right)=w^{d_{\epsilon}^{1}, d_{\epsilon}^{2}}\left(y_{\epsilon}+\xi_{\epsilon}\right)+l\left(\xi_{\epsilon}\right)
$$

Then,

$$
\begin{aligned}
\Phi^{d_{\epsilon}^{1}, d_{\epsilon}^{2}}\left(x_{\epsilon}+\xi_{\epsilon}, y_{\epsilon}+\xi_{\epsilon}\right)= & v^{d_{\epsilon}^{1}, d_{\epsilon}^{2}}\left(x_{\epsilon}+\xi_{\epsilon}\right)-w^{d_{\epsilon}^{1}, d_{\epsilon}^{2}}\left(y_{\epsilon}+\xi_{\epsilon}\right)-\frac{\left|x_{\epsilon}-y_{\epsilon}\right|^{2}}{2 \epsilon} \\
& -\kappa\left[\left\langle x_{\epsilon}+\xi_{\epsilon}\right\rangle^{\bar{m}}+\left\langle y_{\epsilon}+\xi_{\epsilon}\right\rangle^{\bar{m}}\right]
\end{aligned}
$$




$$
\begin{aligned}
\geq & v^{d_{\epsilon}^{1}, d_{\epsilon}^{2}}\left(x_{\epsilon}\right)-w^{d_{\epsilon}^{1}, d_{\epsilon}^{2}}\left(y_{\epsilon}\right)-\frac{\left|x_{\epsilon}-y_{\epsilon}\right|^{2}}{2 \epsilon} \\
& -\kappa\left[\left\langle x_{\epsilon}\right\rangle^{\bar{m}}+\left\langle y_{\epsilon}\right\rangle^{\bar{m}}\right]-2 \kappa\left|\xi_{\epsilon}\right| \\
= & \Phi^{d_{\epsilon}^{1}, d_{\epsilon}^{2}}\left(x_{\epsilon}, y_{\epsilon}\right)-2 \kappa\left|\xi_{\epsilon}\right| \\
\geq & \Phi^{d_{\epsilon}^{1}, d_{\epsilon}^{2}}\left(x_{\epsilon}, y_{\epsilon}\right)-2 \kappa M_{0} .
\end{aligned}
$$

Hence we have

$$
\Phi^{d_{\epsilon}^{1}, d_{\epsilon}^{2}}\left(x_{\epsilon}, y_{\epsilon}\right)-\Phi^{d_{\epsilon}^{1}, d_{\epsilon}^{2}}\left(x_{\epsilon}+\xi_{\epsilon}, y_{\epsilon}+\xi_{\epsilon}\right) \leq 2 \kappa M_{0} .
$$

We will be using this difference to define the new auxiliary function. Observe that $\left|x_{\epsilon}\right|,\left|y_{\epsilon}\right|,\left|\xi_{\epsilon}\right|$ are bounded.

We define the new auxiliary function $\Psi$ by

$$
\begin{aligned}
\Psi^{d^{1}, d^{2}}(x, y)= & \Phi^{d^{1}, d^{2}}(x, y)-\epsilon\left[d\left(x, x_{\epsilon}\right)+d\left(y, y_{\epsilon}\right)\right] \\
& +2(2 \kappa+\epsilon) M_{0} \eta\left(\frac{x-x_{\epsilon}-\xi_{\epsilon}}{\sigma}, \frac{y-y_{\epsilon}-\xi_{\epsilon}}{\sigma}\right),
\end{aligned}
$$

where $\sigma=\sigma(w)$ is the constant coming from Lemma 3.1 and $\eta: \mathbb{R}^{d} \times \mathbb{R}^{d} \rightarrow \mathbb{R}$ is a smooth function with the following properties:

1. $\operatorname{supp}(\eta) \subset B((0,0), 1)$,

2. $0 \leq \eta \leq 1$,

3. $\eta(0,0)=1$ and $\eta<1$ if $(x, y) \neq(0,0)$,

4. $|D \eta| \leq 1$.

Now by the definition of $\Psi$,

$$
\begin{aligned}
\Psi^{d_{\epsilon}^{1}, d_{\epsilon}^{2}}\left(x_{\epsilon}+\xi_{\epsilon}, y_{\epsilon}+\xi_{\epsilon}\right)= & \Phi^{d_{\epsilon}^{1}, d_{\epsilon}^{2}}\left(x_{\epsilon}+\xi_{\epsilon}, y_{\epsilon}+\xi_{\epsilon}\right) \\
& -\epsilon\left[d\left(x_{\epsilon}+\xi_{\epsilon}, x_{\epsilon}\right)+d\left(y_{\epsilon}+\xi_{\epsilon}, y_{\epsilon}\right)\right] \\
& +2(2 \kappa+\epsilon) M_{0} \\
\geq & \Phi^{d_{\epsilon}^{1}, d_{\epsilon}^{2}}\left(x_{\epsilon}, y_{\epsilon}\right)+2 \kappa M_{0}
\end{aligned}
$$

and

$$
\Psi^{d_{\epsilon}^{1}, d_{\epsilon}^{2}}(x, y)=\Phi^{d_{\epsilon}^{1}, d_{\epsilon}^{2}}(x, y) \quad \text { if }\left|x-x_{\epsilon}\right|^{2}+\left|y-y_{\epsilon}\right|^{2} \geq \sigma^{2} .
$$

Hence $\Psi^{d_{\epsilon}^{1}, d_{\epsilon}^{2}}$ attains its maximum in the $\sigma$ ball around $\left(x_{\epsilon}+\xi_{\epsilon}, y_{\epsilon}+\xi_{\epsilon}\right)$ at (say) the point $\left(\hat{x}_{\epsilon}, \hat{y}_{\epsilon}\right)$. By Lemma (3.1), we now know that, for all $\epsilon \in\left(0, \epsilon_{0}\right)$,

$$
w^{d_{\epsilon}^{1}, d_{\epsilon}^{2}}\left(\hat{y}_{\epsilon}\right)<N\left[w^{d_{\epsilon}^{1}, d_{\epsilon}^{2}}\right]\left(\hat{y}_{\epsilon}\right) .
$$

If there is a sequence $\epsilon_{n} \downarrow 0$ such that (3.6) holds for all $n$, then, along this sequence, we proceed to Step 2 with $\Psi^{d^{1}, d^{2}}=\Phi^{d^{1}, d^{2}}$ and $\left(\hat{x}_{\epsilon_{n}}, \hat{y}_{\epsilon_{n}}\right)=\left(x_{\epsilon_{n}}, y_{\epsilon_{n}}\right)$. 
Thus we always have a sequence $\epsilon_{n} \downarrow 0$ along which we have

$$
w^{d_{\epsilon_{n}}^{1}, d_{\epsilon_{n}}^{2}}\left(\hat{y}_{\epsilon_{n}}\right)<N\left[w^{d_{\epsilon_{n}}^{1}, d_{\epsilon_{n}}^{2}}\right]\left(\hat{y}_{\epsilon_{n}}\right)
$$

with $\left(\hat{x}_{\epsilon_{n}}, \hat{y}_{\epsilon_{n}}\right)$ being a maximizer of $\Psi^{d_{\epsilon_{n}}^{1}, d_{\epsilon_{n}}^{2}}$. Since $D^{1} \times D^{2}$ is a finite set, without any loss of generality, we may assume that $\left(d_{\epsilon_{n}}^{1}, d_{\epsilon_{n}}^{2}\right)=\left(d_{0}^{1}, d_{0}^{2}\right)$ for all $n$. In the next step we check what happens to the inequality (3.2) at the maximizer of the new auxiliary function $\Psi^{d_{0}^{1}, d_{0}^{2}}$.

Step 2. Now for each fixed $n$, we proceed as follows. There are two cases either $w^{d_{0}^{1}, d_{0}^{2}}\left(\hat{y}_{\epsilon_{n}}\right)=M_{-}^{d_{0}^{1}, d_{0}^{2}}[w]\left(\hat{y}_{\epsilon_{n}}\right)$ or $w^{d_{0}^{1}, d_{0}^{2}}\left(\hat{y}_{\epsilon_{n}}\right)<M_{-}^{d_{0}^{1}, d_{0}^{2}}[w]\left(\hat{y}_{\epsilon_{n}}\right)$.

If

$$
w^{d_{0}^{1}, d_{0}^{2}}\left(\hat{y}_{\epsilon_{n}}\right)=M_{-}^{d_{0}^{1}, d_{0}^{2}}[w]\left(\hat{y}_{\epsilon_{n}}\right)
$$

then by the definition of $M_{-}^{d_{0}^{1}, d_{0}^{2}}$, there exists $d_{n_{1}}^{2} \in D^{2}$ such that

$$
w^{d_{0}^{1}, d_{0}^{2}}\left(\hat{y}_{\epsilon_{n}}\right)=w^{d_{0}^{1}, d_{n_{1}}^{2}}\left(\hat{y}_{\epsilon_{n}}\right)+c^{2}\left(d_{0}^{2}, d_{n_{1}}^{2}\right) .
$$

We know that

$$
v^{d_{0}^{1}, d_{0}^{2}}\left(\hat{x}_{\epsilon_{n}}\right) \leq v^{d_{0}^{1}, d_{n_{1}}^{2}}\left(\hat{x}_{\epsilon_{n}}\right)+c^{2}\left(d_{0}^{2}, d_{n_{1}}^{2}\right) .
$$

Hence,

$$
\begin{aligned}
\Psi^{d_{0}^{1}, d_{0}^{2}}\left(\hat{x}_{\epsilon_{n}}, \hat{y}_{\epsilon_{n}}\right)-\Psi^{d_{0}^{1}, d_{n_{1}}^{2}}\left(\hat{x}_{\epsilon_{n}}, \hat{y}_{\epsilon_{n}}\right) \leq & v^{d_{0}^{1}, d_{n_{1}}^{2}}\left(\hat{x}_{\epsilon_{n}}\right)+c^{2}\left(d_{0}^{2}, d_{n_{1}}^{2}\right) \\
& -w^{d_{0}^{1}, d_{n_{1}}^{2}}\left(\hat{y}_{\epsilon_{n}}\right)-c^{2}\left(d_{0}^{2}, d_{n_{1}}^{2}\right) \\
\leq & 0 .
\end{aligned}
$$

Hence we get

$$
\Psi^{d_{0}^{1}, d_{0}^{2}}\left(\hat{x}_{\epsilon_{n}}, \hat{y}_{\epsilon_{n}}\right) \leq \Psi^{d_{0}^{1}, d_{n_{1}}^{2}}\left(\hat{x}_{\epsilon_{n}}, \hat{y}_{\epsilon_{n}}\right) .
$$

Now if strict inequality holds in $w^{d_{0}^{1}, d_{n_{1}}^{2}}\left(\hat{y}_{\epsilon_{n}}\right) \leq M_{-}^{d_{0}^{1}, d_{n_{1}}^{2}}[w]\left(\hat{y}_{\epsilon_{n}}\right)$, then we are done; else we repeat the above argument and get $d_{n_{2}}^{2} \in \bar{D}^{2}$ such that

$$
w^{d_{0}^{1}, d_{n_{1}}^{2}}\left(\hat{y}_{\epsilon_{n}}\right)=w^{d_{0}^{1}, d_{n_{2}}^{2}}\left(\hat{y}_{\epsilon_{n}}\right)+c^{2}\left(d_{n_{1}}^{2}, d_{n_{2}}^{2}\right) .
$$

Now

$$
\begin{aligned}
w^{d_{0}^{1}, d_{n_{2}}^{2}\left(\hat{y}_{\epsilon_{n}}\right)} & =w^{d_{0}^{1}, d_{n_{1}}^{2}}\left(\hat{y}_{\epsilon_{n}}\right)-c^{2}\left(d_{n_{1}}^{2}, d_{n_{2}}^{2}\right) \\
& \geq w^{d_{0}^{1}, d_{n_{1}}^{2}}\left(\hat{y}_{\epsilon_{n}}\right)-c_{0}^{2} \\
& \geq w^{d_{0}^{1}, d_{0}^{2}}\left(\hat{y}_{\epsilon_{n}}\right)-2 c_{0}^{2} .
\end{aligned}
$$


Proceeding in similar fashion, after finitely many steps, boundedness of $w$ will be contradicted and hence for some $d_{n}^{2}=d_{n_{j}}^{2} \in D^{2}$, we must have

$$
w^{d_{0}^{1}, d_{n}^{2}}\left(\hat{y}_{\epsilon_{n}}\right)<M_{-}^{d_{0}^{1}, d_{n}^{2}}[w]\left(\hat{y}_{\epsilon_{n}}\right)
$$

and

$$
\Psi^{d_{0}^{1}, d_{0}^{2}}\left(\hat{x}_{\epsilon_{n}}, \hat{y}_{\epsilon_{n}}\right) \leq \Psi^{d_{0}^{1}, d_{n}^{2}}\left(\hat{x}_{\epsilon_{n}}, \hat{y}_{\epsilon_{n}}\right) .
$$

On the other hand, if

$$
w^{d_{0}^{1}, d_{0}^{2}}\left(\hat{y}_{\epsilon_{n}}\right)<M_{-}^{d_{0}^{1}, d_{0}^{2}}[w]\left(\hat{y}_{\epsilon_{n}}\right),
$$

then we proceed by taking $d_{n}^{2}=d_{0}^{2}$.

Step 3. For each fixed $n$, we proceed as follows: If

$$
v^{d_{0}^{1}, d_{n}^{2}}\left(\hat{x}_{\epsilon_{n}}\right)=M_{+}^{d_{0}^{1}, d_{n}^{2}}[v]\left(\hat{x}_{\epsilon_{n}}\right),
$$

then we proceed as in Step 2 and obtain $d_{n}^{1}=d_{n_{i}}^{1} \in D^{1}$ such that

$$
v^{d_{n}^{1}, d_{n}^{2}}\left(\hat{x}_{\epsilon_{n}}\right)>M_{+}^{d_{n}^{1}, d_{n}^{2}}[v]\left(\hat{x}_{\epsilon_{n}}\right)
$$

and

$$
\Psi^{d_{0}^{1}, d_{n}^{2}}\left(\hat{x}_{\epsilon_{n}}, \hat{y}_{\epsilon_{n}}\right) \leq \Psi^{d_{n}^{1}, d_{n}^{2}}\left(\hat{x}_{\epsilon_{n}}, \hat{y}_{\epsilon_{n}}\right) .
$$

If

$$
v^{d_{0}^{1}, d_{n}^{2}}\left(\hat{x}_{\epsilon_{n}}\right)=M_{+}^{d_{0}^{1}, d_{n}^{2}}[v]\left(\hat{x}_{\epsilon_{n}}\right),
$$

then we proceed by taking $d_{n}^{1}=d_{0}^{1}$.

Thus, for every $n,\left(\hat{x}_{\epsilon_{n}}, \hat{y}_{\epsilon_{n}}\right)$ is a maximizer of $\Psi^{d_{n}^{1}, d_{n}^{2}}$ and

$$
\begin{gathered}
w^{d_{n}^{1}, d_{n}^{2}}\left(\hat{y}_{\epsilon_{n}}\right)<N\left[w^{d_{n}^{1}, d_{n}^{2}}\right]\left(\hat{y}_{\epsilon_{n}}\right), \\
w^{d_{n}^{1}, d_{n}^{2}}\left(\hat{y}_{\epsilon_{n}}\right)<M_{-}^{d_{n}^{1}, d_{n}^{2}}[w]\left(\hat{y}_{\epsilon_{n}}\right), \\
v^{d_{n}^{1}, d_{n}^{2}}\left(\hat{x}_{\epsilon_{n}}\right)>M_{+}^{d_{n}^{1}, d_{n}^{2}}[v]\left(\hat{x}_{\epsilon_{n}}\right) .
\end{gathered}
$$

Also by using (3.11) and (3.12), we have that

$$
\Psi^{d_{0}^{1}, d_{0}^{2}}\left(\hat{x}_{\epsilon_{n}}, \hat{y}_{\epsilon_{n}}\right) \leq \Psi^{d_{n}^{1}, d_{n}^{2}}\left(\hat{x}_{\epsilon_{n}}, \hat{y}_{\epsilon_{n}}\right) .
$$

Now we define test functions $\phi_{1 n}$ and $\phi_{2 n}$ as follows:

$$
\begin{aligned}
\phi_{1 n}(x)= & w^{d_{n}^{1}, d_{n}^{2}}\left(\hat{y}_{\epsilon_{n}}\right)+\frac{\left|x-\hat{y}_{\epsilon_{n}}\right|^{2}}{2 \epsilon_{n}}+\kappa\left[\langle x\rangle^{\bar{m}}+\left\langle\hat{y}_{\epsilon_{n}}\right\rangle^{\bar{m}}\right] \\
& -2\left(2 \kappa+\epsilon_{n}\right) M_{0} \eta\left(\frac{x-x_{\epsilon_{n}}-\xi_{\epsilon_{n}}}{\sigma}, \frac{\hat{y}_{\epsilon_{n}}-y_{\epsilon_{n}}-\xi_{\epsilon_{n}}}{\sigma}\right), \\
\phi_{2 n}(y)= & v^{d_{n}^{1}, d_{n}^{2}}\left(\hat{x}_{\epsilon_{n}}\right)-\frac{\left|\hat{x}_{\epsilon_{n}}-y\right|^{2}}{2 \epsilon_{n}}-\kappa\left[\left\langle\hat{x}_{\epsilon_{n}}\right\rangle^{\bar{m}}+\langle y\rangle^{\bar{m}}\right] \\
& +2\left(2 \kappa+\epsilon_{n}\right) M_{0} \eta\left(\frac{\hat{x}_{\epsilon_{n}}-x_{\epsilon_{n}}-\xi_{\epsilon_{n}}}{\sigma}, \frac{y-y_{\epsilon_{n}}-\xi_{\epsilon_{n}}}{\sigma}\right) .
\end{aligned}
$$


Observe that

$$
\begin{aligned}
D \phi_{1 n}\left(\hat{x}_{\epsilon_{n}}\right)= & \frac{\hat{x}_{\epsilon_{n}}-\hat{y}_{\epsilon_{n}}}{\epsilon_{n}}+\kappa \bar{m}\left\langle\hat{x}_{\epsilon_{n}}\right\rangle^{m-2} \hat{x}_{\epsilon_{n}} \\
& -\frac{2\left(2 \kappa+\epsilon_{n}\right) M_{0}}{\sigma} D_{x} \eta\left(\frac{\hat{x}_{\epsilon_{n}}-x_{\epsilon_{n}}-\xi_{\epsilon_{n}}}{\sigma}, \frac{\hat{y}_{\epsilon_{n}}-y_{\epsilon_{n}}-\xi_{\epsilon_{n}}}{\sigma}\right), \\
D \phi_{2 n}\left(\hat{y}_{\epsilon_{n}}\right)= & \frac{\hat{x}_{\epsilon_{n}}-\hat{y}_{\epsilon_{n}}}{\epsilon_{n}}-\kappa \bar{m}\left\langle\hat{y}_{\epsilon_{n}}\right\rangle^{m-2} \hat{y}_{\epsilon_{n}} \\
& +\frac{2\left(2 \kappa+\epsilon_{n}\right) M_{0}}{\sigma} D_{y} \eta\left(\frac{\hat{x}_{\epsilon_{n}}-x_{\epsilon_{n}}-\xi_{\epsilon_{n}}}{\sigma}, \frac{\hat{y}_{\epsilon_{n}}-y_{\epsilon_{n}}-\xi_{\epsilon_{n}}}{\sigma}\right) .
\end{aligned}
$$

Note that $v^{d_{n}^{1}, d_{n}^{2}}-\phi_{1 n}$ attains its maximum at $\hat{x}_{\epsilon_{n}}$ and $w^{d_{n}^{1}, d_{n}^{2}}-\phi_{2 n}$ attains its minimum at $\hat{y}_{\epsilon_{n}}$. Hence, as $\epsilon_{n} \rightarrow 0$, we have

$$
\begin{aligned}
\lambda\left[v^{d_{n}^{1}, d_{n}^{2}}\left(\hat{x}_{\epsilon_{n}}\right)-w^{d_{n}^{1}, d_{n}^{2}}\left(\hat{y}_{\epsilon_{n}}\right)\right] \leq & H_{+}^{d_{n}^{1}, d_{n}^{2}}\left(\hat{y}_{\epsilon_{n}}, D \phi_{2 n}\left(\hat{y}_{\epsilon_{n}}\right)\right)-H_{+}^{d_{n}^{1}, d_{n}^{2}}\left(\hat{x}_{\epsilon_{n}}, D \phi_{1 n}\left(\hat{x}_{\epsilon_{n}}\right)\right)+o(1) \\
\leq & L\left|\hat{x}_{\epsilon_{n}}-\hat{y}_{\epsilon_{n}}\right|\left(1+\left|\frac{\hat{x}_{\epsilon_{n}}-\hat{y}_{\epsilon_{n}}}{\epsilon_{n}}\right|\right)+o(1) \\
& +\|f\|_{\infty}\left[\kappa \bar{m}\left(\left\langle\hat{x}_{\epsilon_{n}}\right\rangle^{\bar{m}-1}+\left\langle\hat{y}_{\epsilon_{n}}\right\rangle^{\bar{m}-1}\right)+\frac{4(2 \kappa+\epsilon) M_{0}}{\sigma}\right] .
\end{aligned}
$$

Note that we have used $|y| \leq\left(1+|y|^{2}\right)^{1 / 2}=\langle y\rangle$ to get the above inequality. Now as $\bar{m}-1<0$, it follows that

$$
\begin{aligned}
& v^{d_{n}^{1}, d_{n}^{2}}\left(\hat{x}_{\epsilon_{n}}\right)-w^{d_{n}^{1}, d_{n}^{2}}\left(\hat{y}_{\epsilon_{n}}\right) \leq \frac{L}{\lambda}\left|\hat{x}_{\epsilon_{n}}-\hat{y}_{\epsilon_{n}}\right|+\frac{L}{\lambda} \frac{\left|\hat{x}_{\epsilon_{n}}-\hat{y}_{\epsilon_{n}}\right|^{2}}{\epsilon_{n}}+o(1) \\
& +\frac{2\|f\|_{\infty} \kappa \bar{m}}{\lambda}+\frac{4\|f\|_{\infty} \kappa M_{0}}{\sigma \lambda} \\
& \leq \frac{2\|f\|_{\infty} \kappa \bar{m}}{\lambda}+\frac{4\|f\|_{\infty} \kappa M_{0}}{\sigma \lambda}+o(1) ; \text { as } \epsilon_{n} \downarrow 0 .
\end{aligned}
$$

For any $x \in \mathbb{R}^{d}$ and $\left(d^{1}, d^{2}\right) \in D^{1} \times D^{2}$,

$$
\begin{aligned}
v^{d^{1}, d^{2}}(x)-w^{d^{1}, d^{2}}(x)-2 \kappa\langle x\rangle^{\bar{m}} & =\Phi^{d^{1}, d^{2}}(x, x) \\
& \leq \Phi^{d_{0}^{1}, d_{0}^{2}}\left(x_{\epsilon_{n}}, y_{\epsilon_{n}}\right)+\epsilon_{n}\left[d\left(x, x_{\epsilon_{n}}\right)+d\left(x, y_{\epsilon_{n}}\right)\right] \\
& \leq \Psi^{d_{0}^{1}, d_{0}^{2}}\left(x_{\epsilon_{n}}, y_{\epsilon_{n}}\right)+o(1) ; \text { as } \epsilon_{n} \downarrow 0 .
\end{aligned}
$$

Since $\Psi^{d_{0}^{1}, d_{0}^{2}}$ attains its maximum at $\left(\hat{x}_{\epsilon_{n}}, \hat{y}_{\epsilon_{n}}\right)$ in the $\sigma$ ball around $\left(x_{\epsilon_{n}}+\xi_{\epsilon_{n}}, y_{\epsilon_{n}}+\xi_{\epsilon_{n}}\right)$, for an appropriate constant $C$, we have

$$
\begin{aligned}
\Psi^{d_{0}^{1}, d_{0}^{2}}\left(x_{\epsilon_{n}}, y_{\epsilon_{n}}\right) & \leq \Psi^{d_{0}^{1}, d_{0}^{2}}\left(\hat{x}_{\epsilon_{n}}, \hat{y}_{\epsilon_{n}}\right) \\
& \leq \Psi^{d_{n}^{1}, d_{n}^{2}}\left(\hat{x}_{\epsilon_{n}}, \hat{y}_{\epsilon_{n}}\right) \text { using (3.16) }
\end{aligned}
$$




$$
\begin{aligned}
& \leq v^{d_{n}^{1}, d_{n}^{2}}\left(\hat{x}_{\epsilon_{n}}\right)-w^{d_{n}^{1}, d_{n}^{2}}\left(\hat{y}_{\epsilon_{n}}\right)+C \kappa+o(1) ; \text { as } \epsilon_{n} \downarrow 0 \\
& \leq \frac{2\|f\|_{\infty} \kappa \bar{m}}{\lambda}+\frac{4\|f\|_{\infty} \kappa M_{0}}{\sigma \lambda}+C \kappa+o(1) ; \text { as } \epsilon_{n} \downarrow 0 .
\end{aligned}
$$

Hence we get

$$
\begin{aligned}
& v^{d^{1}, d^{2}}(x)-w^{d^{1}, d^{2}}(x)-2 \kappa\langle x\rangle^{\bar{m}} \\
& \quad \leq \frac{2\|f\|_{\infty} \kappa \bar{m}}{\lambda}+\frac{4\|f\|_{\infty} \kappa M_{0}}{\sigma \lambda}+C \kappa+o(1) ; \text { as } \epsilon_{n} \downarrow 0 .
\end{aligned}
$$

Now let $\epsilon_{n} \downarrow 0$ and then $\kappa \downarrow 0$, to obtain

$$
v^{d^{1}, d^{2}}(x)-w^{d^{1}, d^{2}}(x) \leq 0 .
$$

This completes the proof of uniqueness for (HJI+).

The above uniqueness result holds true if one is the viscosity solution and the other is an approximate viscosity solution. This is the content of the next theorem.

Theorem 3.4. Assume (A1) and (A2). Let $v$ and $w \in \mathrm{BUC}\left(\mathbb{E} ; \mathbb{R}^{m_{1} \times m_{2}}\right)$. Let $v$ be a viscosity solution of $(\mathrm{HJI}+)$ (resp. (HJI-)) and $w$ be an approximate viscosity solution of $(\mathrm{HJI}+)($ resp. $(H J I-))$. Then $v=w$.

Proof. The proof is similar to that of the previous theorem. The only change here is in (3.5). Since $w$ is an approximate viscosity solution, one gets

$$
\begin{aligned}
& \lambda w^{d_{\epsilon}^{1}, d_{\epsilon}^{2}}\left(y_{\epsilon}\right)+D_{A}^{+} \Phi_{2}\left(y_{\epsilon}\right)+H_{+}^{d_{\epsilon}^{1}, d_{\epsilon}^{2}}\left(y_{\epsilon}, \frac{x_{\epsilon}-y_{\epsilon}}{\epsilon}-\kappa \bar{m}\left\langle y_{\epsilon}\right\rangle^{\bar{m}-2} y_{\epsilon}\right) \\
& \geq-\epsilon C_{R},
\end{aligned}
$$

where $R=\sup _{\epsilon>0}\left\|y_{\epsilon}\right\|$. Note that, for fixed $\kappa, R<\infty$ and hence

$$
\epsilon C_{R}=o(1) \quad \text { as } \epsilon \rightarrow 0 \text {. }
$$

Once we have this inequality (instead of (3.5)), we mimic all other arguments in the proof of the previous theorem.

Now we can prove our main result stated in $\S 1$, namely Theorem 1.5.

Proof of Theorem 1.5. Under the Isaacs min-max condition, (HJI-) and (HJI+) coincide. Let us denote this equation by (HJI). As in [2], by Perron's method, we can prove an existence of a viscosity solution for (HJI) in BUC $\left(\mathbb{E}, \mathbb{R}^{m_{1} \times m_{2}}\right)$, the class of bounded uniformly continuous functions. Let $W$ be any such viscosity solution. Now, by Theorem 2.7 we know that lower and upper value functions, $V_{-}$and $V_{+}$are approximate viscosity solutions of (HJ). Therefore, by Theorem 3.4, $V_{-}=W=V_{+}$. This proves the main result. 


\section{Conclusions}

We have studied two-person zero-sum differential games with hybrid controls in infinite dimension. The minimizing player uses continuous, switching, and impulse controls whereas the maximizing player uses continuous and switching controls. The dynamic programming principle for lower and upper value functions is proved and using this we have established the existence and uniqueness of the value under Isaacs min-max condition.

For finite dimensional problems, similar result has been obtained by Yong [8] under two additional assumptions:

(Y1) Cheaper switching cost condition

$$
\min _{\bar{d}^{2} \neq d^{2}} c^{2}\left(d^{2}, \bar{d}^{2}\right)=: c_{0}^{2}<l_{0}=\inf _{\xi \in K} l(\xi) .
$$

\section{(Y2) Nonzero loop switching cost condition}

For any loop $\left\{\left(d_{i}^{1}, d_{i}^{2}\right)\right\}_{i=1}^{j} \subset D^{1} \times D^{2}$, with the property that

$$
\begin{aligned}
& j \leq m_{1} m_{2}, d_{j+1}^{1}=d_{1}^{1}, d_{j+1}^{2}=d_{1}^{2} ; \\
& \text { either } d_{i+1}^{1}=d_{i}^{1}, \text { or } d_{i+1}^{2}=d_{i}^{2} \quad \forall 1 \leq i \leq j .
\end{aligned}
$$

It holds that

$$
\sum_{i=1}^{j} c^{1}\left(d_{i}^{1}, d_{i+1}^{1}\right)-\sum_{i=1}^{j} c^{2}\left(d_{i}^{2}, d_{i+1}^{2}\right) \neq 0 .
$$

Thus our result not only extends the work of [8] to infinite dimensions but also proves the uniqueness of the viscosity solutions of upper and lower SQVI without the above two conditions (Y1) and (Y2). Also we have shown that under Isaacs' min-max condition, the game has a value. Moreover, we have given explicit formulation of dynamic programming principle for hybrid differential games and have also proved it which is not done in [8].

\section{Acknowledgement}

The authors wish to thank M K Ghosh for suggesting the problem and for several useful discussions. They also thank M K Ghosh and Mythily Ramaswamy for carefully reading the manuscript and for useful suggestions. Financial support from NBHM is gratefully acknowledged.

\section{References}

[1] Bardi M and Capuzzo-Dolcetta I, Optimal control and viscosity solutions of HamiltonJacobi-Bellman equations (Birkhauser) (1997)

[2] Crandall M G and Lions P L, Hamilton-Jacobi equations in infinite dimensions, Part VI: Nonlinear A and Tataru's method refined, Evolution Equations, Control Theory and Biomathematics, Lecture Notes in Pure and Applied Mathematics, Dekker 155 (1994) 51-89

[3] Evans L C and Souganidis P E, Differential games and representation formulas for Hamilton-Jacobi equations, Indiana Univ. Math. J. 33 (1984) 773-797 
[4] Kocan M, Soravia P and Swiech A, On differential games for infinite-dimensional systems with nonlinear, unbounded operators, J. Math. Anal. Appl. 211 (1997) 395-423

[5] Pazy A, Semigroups of linear operators and applications to partial differential equations, Applied Mathematical Sciences 44 (Springer-Verlag) (1989)

[6] Yong J, Differential games with switching strategies, J. Math. Anal. Appl. 145 (1990) 455-469

[7] Yong J, A zero-sum differential game in a finite duration with switching strategies, SIAM J. Control Optim. 28 (1990) 1234-1250

[8] Yong J, Zero-sum differential games involving impulse controls, Appl. Math. Optim. 29 (1990) 243-261 